\title{
Convergence analysis for a smeared crack approach in brittle fracture
}

\author{
MATTEO NEgRI ${ }^{\dagger}$ \\ Dipartimento di Matematica, Università degli Studi di Pavia, \\ Via Ferrata 1, 27100 Pavia, Italy
}

[Received 21 October 2005 and in revised form 10 October 2006]

\begin{abstract}
Our analysis focuses on the mechanical energies involved in the propagation of fractures: the elastic energy, stored in the bulk, and the fracture energy, concentrated in the crack. We consider a finite element model based on a smeared crack approach: the fracture is approximated geometrically by a stripe of elements and mechanically by a softening constitutive law. We define in this way a discrete free energy $G_{h}$ ( $h$ being the element size) which accounts for both elastic displacements and fractures. Our main interest is the behaviour of $G_{h}$ as $h \rightarrow 0$. We prove that, for a suitable choice of the (mesh dependent) constitutive law, $G_{h}$ converges to a limit functional $G_{\phi}$ with a positive (anisotropic) term concentrated on the crack. We discuss the mesh bias and compute it explicitly in the case of a structured triangulation.
\end{abstract}

\section{Introduction}

Smeared cracking is a well known finite element model for simulating strain localization phenomena, including the propagation of fractures. Starting from the ideas of Rashid [27] this technique has attracted much attention in the field of computational mechanics, being continuously studied and improved (see for instance [4], [28] and the references therein). Besides academic research, smeared cracking has also been used for real life applications and has been implemented in commercial software for structural mechanics.

In its "classical" form, the idea consists basically in replacing the fracture with a band of finite elements (see Figure 4). This geometrical approximation is then accompanied with a softening constitutive law of damage type. These two aspects, geometrical and constitutive, characterize the different versions of this approach and have been the main source of investigation and discussion. Different authors have noted that, for some choices of the softening law, when the size of the triangulation becomes too small the model gives a wrong mechanical response where no dissipation occurs. Moreover, it has often been pointed out that the geometry of the mesh introduces an artificial bias which may affect strongly the direction of propagation. These points have been treated and often circumvented in different ways, for instance by means of constitutive laws depending on the mesh size [25], [26] and/or by a non-local approach [19].

Apart from physical considerations, for which we refer to the specific engineering literature, to our knowledge a mathematical treatment of the smeared cracking model has been missing. The model examined in this work is quite simple, compared with the complexity of some actual engineering implementation, nonetheless it catches clearly two main aspects, i.e. scaling and mesh bias which are fundamental in computational mechanics [21], [18]. The importance of these features is well explained by Oliver [25]: "A common feature of these models is their 'non-objectivity'

†E-mail: matteo.negri@unipv.it 
with respect to the size of the finite elements mesh when standard finite elements of $C^{0}$ continuity are used. Objectivity can be achieved by modifying the constitutive law and making it depend on mesh size by introducing a parameter called 'crack band width' or 'characteristic length'. For fairly regular meshes this parameter is frequently determined in an intuitive way which, however, is difficult to generalize in a formal manner for irregular meshes and arbitrary crack directions." We will see (in $\$ 2$ and $\$ 3$ ) that our analysis gives a possible answer to the questions raised by Oliver.

The point of view adopted here is purely based on the energies related to fracture propagation and mathematically relies strongly on the theories of $S B D$ functions [2] and $\Gamma$-convergence [15]. Without entering into technical details, let us explain briefly the main result, starting from the continuum model. We consider a reference two-dimensional domain $\Omega$ with in-plane, possibly discontinuous, deformations. Denoting by $u$ the displacement field, the fracture will always be identified with the set $J(u)$ where $u$ is discontinuous. We will consider a brittle material and assume cracking to be governed by Griffith's model. Thus, the energy concentrated on the fracture will be of the form $\gamma|J(u)|$ (where $|\cdot|$ denotes the length measure and $\gamma$ is a material parameter) while the energy stored in the bulk will be the linearized elastic energy $W^{e}(\varepsilon(u)$ ) (where $\varepsilon(u)$ is the symmetrized gradient of $u$ ). Hence, in the continuum setting, the free energy will be of the form

$$
G(u)=\int_{\Omega} W^{e}(\varepsilon(u)) \mathrm{d} x+\gamma|J(u)| .
$$

Fracture propagation can be modelled as an evolution associated with such an energy. Recently, much attention has been payed to this subject, in particular as regards the quasi-static propagation based on minimizing movements, i.e. on sequences of minimizers of $G$. We refer the interested reader to [17], [11], [16] and [7]. This aspect is beyond the scope of this paper. We limit our analysis to giving a rigorous approximation of $G$, in terms of a smeared cracking model, which, however, is a basic problem for time dependent models.

Our finite element model is defined in the prototype case of piecewise linear elements on a structured mesh $\boldsymbol{T}_{h}$; the energy is simply of the form

$$
G_{h}\left(u_{h}\right)=\int_{\Omega} W_{h}\left(\varepsilon\left(u_{h}\right)\right) .
$$

The density $W_{h}$ is defined by means of a damage constitutive law of the kind

$$
\frac{\partial W_{h}}{\partial \varepsilon}=\left[1-d\left(h W^{e}(\varepsilon)\right)\right] \sigma=\left[1-d\left(h W^{e}(\varepsilon)\right)\right] \frac{\partial W^{e}}{\partial \varepsilon},
$$

for a suitable choice of the damage function $d$ which governs the transition between the elastic and fracture regimes. We remark that $d$ depends on the elastic energy density through a rescaling factor $h$ which is crucial for our convergence result. We will see that this factor plays the role of the characteristic length in [25]. Denoting by $f$ a primitive of $1-d$ we can clearly write

$$
W_{h}(\varepsilon)=\frac{1}{h} f\left(h W^{e}(\varepsilon)\right) .
$$

We remark that a similar approach has been widely used for the approximation of the MumfordShah functional both for adaptive [13], [6] and structured triangulations [22] while it has been employed in a non-local form for the propagation of fractures [7]. 
Our main interest is the convergence of $G_{h}$ as the element size $h$ tends to zero, or, in other terms, to understand when $G_{h}$ is an approximation of $G$. More precisely, we prove that $G_{h} \Gamma$-converges (as $h \rightarrow 0$ tends to zero and with respect to the $L^{1}$-topology) to an energy of the form

$$
G_{\phi}(u)=\int_{\Omega \backslash J(u)} W^{e}(\varepsilon(u)) \mathrm{d} x+\gamma \int_{J(u)} \phi(v) \mathrm{d} s,
$$

which reminds a similar formula obtained in [22] for the Mumford-Shah functional. The density $\phi$ represents the mesh bias due to the geometry of the triangulation $\boldsymbol{T}_{h}$ for every possible crack direction. Indeed $\phi$ depends on the normal vector $v$ to the set $J(u)$ and thus it depends on the orientation of the fracture. This term is explicitly computed in the case of structured triangulations (see $\$ 3$. Unfortunately, for a general unstructured mesh it is not possible to characterize it in a precise way since in general a unique $\Gamma$-limit is not possible. Finally, in the case of a scale independent law we can show that the limit energy $G$ would be identically zero. This fact explains, in mathematical terms, why in some cases it has been observed that the dissipation was almost vanishing for small mesh sizes.

From the technical point of view the main difficulty lies in the $\Gamma$-liminf inequality. Its proof requires first a suitable application of the slicing technique, to take into account the geometry of the triangulation, and then a "strange" one-dimensional estimate, depending at the same time on two neighbouring sections. We recall that a convergence result of this kind, with a slightly different fracture energy, has been obtained also in [1] using an "atomistic" approach by finite differences. Generalizations of our convergence result to the case of non-local and cohesive models (or to the case of other non-local operators) are still quite hard due to some technical problems with symmetrized gradients. These topics will be the subject of future investigations and forthcoming papers [20]. Hopefully, these mathematical results, combined with comparative studies [19], will provide a deeper understanding of softening models and some useful properties for the design of robust finite element simulations.

\section{Anisotropic limit for structured triangulations}

Let $\Omega$ be an open bounded Lipschitz set in $\mathbb{R}^{2}$ and let $\left\{\boldsymbol{T}_{h}\right\}$ (for $h>0$ ) be a regular family of structured triangulations. For simplicity we will consider the prototype mesh represented in Figure 1 which is defined on the grid $(h / \sqrt{2}) \mathbb{Z}^{2}$ (in such a way that the diameter of the elements is $h$ ). We denote by $V_{h}\left(\Omega, \mathbb{R}^{2}\right)$ the finite element set of piecewise affine functions on $\boldsymbol{T}_{h}$ taking values in $\mathbb{R}^{2}$ and uniformly bounded in $L^{\infty}$ by some positive constant $k$ which can be arbitrarily large. Note that

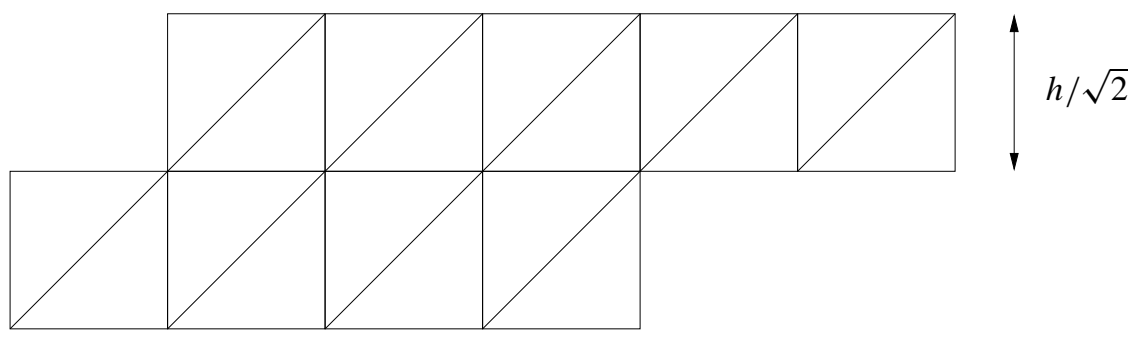

FIG. 1. The structure of the triangulation $\boldsymbol{T}_{h}$. 
this constraint is purely technical and avoids truncations of the displacement field. We will always write $V_{h}$ instead of $V_{h}\left(\Omega, \mathbb{R}^{2}\right)$ and similarly for other function spaces.

Let $\varepsilon=\varepsilon(u)$ be the symmetrized gradient and consider the linearized elastic energy density

$$
W^{e}(\varepsilon(u))=\mu|\varepsilon(u)|^{2}+\frac{\lambda}{2}|\operatorname{tr} \varepsilon(u)|^{2},
$$

where $\mu$ and $\lambda$ are material parameters (the Lamé constants). Let $f:[0,+\infty) \rightarrow \mathbb{R}$ be an increasing, continuous function such that

$$
\lim _{t \rightarrow 0^{+}} \frac{f(t)}{t}=1, \quad \lim _{t \rightarrow+\infty} f(t)=\gamma .
$$

Let $f_{h}(t)=f(h t) / h$ be a rescaling of $f$ and define

$$
W_{h}(\varepsilon(u))=f_{h}\left(W^{e}(\varepsilon(u))\right) .
$$

The discrete energy is then given by

$$
G_{h}\left(u_{h}\right)= \begin{cases}\int_{\Omega} W_{h}\left(\varepsilon\left(u_{h}\right)\right) \mathrm{d} x, & u_{h} \in V_{h}, \\ +\infty, & u_{h} \in L^{1} \backslash V_{h} .\end{cases}
$$

The convergence result for $G_{h}$ is summarized in the following theorem.

THEOREM 2.1 The functionals $G_{h} \Gamma$-converge, with respect to the $L^{1}$-topology, to the functional

$$
G(u)= \begin{cases}\int_{\Omega \backslash J(u)} W^{e}(\varepsilon(u)) \mathrm{d} x+\gamma \int_{J(u)} \phi(v) \mathrm{d} s, & u \in S B D^{2},\|u\|_{L^{\infty}}<k, \\ +\infty, & \text { otherwise in } L^{1} .\end{cases}
$$

The density $\phi$, appearing in the length term and depending on the normal $v$ to $J(u)$, represents the mesh bias and depends only on the discrete geometry of the triangulations (for more details we refer to Section 3. For simplicity we denote by $d s$ the one-dimensional Hausdorff measure, i.e. the length measure.

Finally, if $u_{h}$ is a family in $V_{h}$ which is equibounded in energy, namely with $G_{h}\left(u_{h}\right) \leqslant c$, then $u_{h}$ is strongly precompact in $L^{p}$ for every $1 \leqslant p<+\infty$.

REMARK 2.2 Note that the function $f$, and its rescaling $f_{h}$, introduce in the discrete energy a local softening which depends on the size $h$ of the elements. Scaling the constitutive law in this way is necessary to recover in the limit the correct fracture energy. Indeed, consider the scale independent energy

$$
E_{h}(u)= \begin{cases}\int_{\Omega} f\left(W^{e}(\varepsilon(u))\right) \mathrm{d} x & \text { if } u \in V_{h}, \\ +\infty & \text { if } u \in L^{1} \backslash V_{h} .\end{cases}
$$

Then $E_{h}$ converges to a functional $E$ which is identically zero. This fact has been observed numerically by many authors and usually referred to as non-objectivity of the triangulation: for 
$h \rightarrow 0$ the deformation localizes in narrow bands while the energy decreases to zero. From the theoretical point of view this effect can be explained in terms of relaxation. More precisely: for $u \in H^{1}$ let $u_{h} \in V_{h}$ converge to $u$ with respect to the $H^{1}$-norm. As $f\left(W^{e}(M)\right)$ is a Lipschitz function from $\mathbb{R}^{2 \times 2}$ (the space of $2 \times 2$ matrices) into $\mathbb{R}$ we get

$$
\left|\int_{\Omega}\left[f\left(W^{e}\left(\varepsilon\left(u_{h}\right)\right)\right)-f\left(W^{e}(\varepsilon(u))\right)\right] \mathrm{d} x\right| \leqslant L \int_{\Omega}\left|\varepsilon\left(u_{h}\right)-\varepsilon(u)\right|^{2} \mathrm{~d} x .
$$

Thus

$$
\limsup _{h \rightarrow 0} E_{h}\left(u_{h}\right) \leqslant \int_{\Omega} f\left(W^{e}(\varepsilon(u))\right) \mathrm{d} x .
$$

Denote by $\tilde{E}$ the functional

$$
\tilde{E}(u)= \begin{cases}\int_{\Omega} f\left(W^{e}(\varepsilon(u))\right) \mathrm{d} x & \text { if } u \in H^{1}, \\ +\infty & \text { if } u \in L^{1} \backslash H^{1} .\end{cases}
$$

Note that, $f$ being bounded from above, $\tilde{E}$ is not lower semicontinuous with respect to the $L^{1}$ topology, and its lower semicontinuous envelope is identically zero. Since the $\Gamma$-limit $E$ is lower semicontinuous, from (4) it follows that $E$ is identically zero as well.

The main consequence of $\Gamma$-convergence is the convergence of minimizers. For instance, denoting by $\partial_{D} \Omega$ a subset of the boundary $\partial \Omega$, let us consider the problem

$$
\min \left\{G_{\phi}(u): u \in S B D^{2} \text { and } u=g \text { in } \partial_{D} \Omega\right\},
$$

which is basically the incremental problem of [17]. Note that, for simplicity, we do not bother with the precise definition of the boundary condition, which should allow fractures on $\partial_{D} \Omega$ (see e.g. [16]), nor with the irreversibility constraints, which enter in the quasi-static evolution. The direct method of the calculus of variations yields the existence of a minimizer in $S B D^{2}$. Now, denoting by $g_{h}$ the Lagrange interpolation of $g$, we consider the discrete problems

$$
\min \left\{G_{h}\left(u_{h}\right): u_{h} \in V_{h} \text { and } u_{h}=g_{h} \text { in } \partial_{D} \Omega\right\}
$$

and a family $\left\{u_{h}\right\}$ of minimizers. Then $G_{h}\left(u_{h}\right) \leqslant c$ and by Theorem 2.1 it follows that $u_{h}$ is precompact in $L^{1}$. Hence, there exists a subsequence $u_{h_{n}}$ of $u_{h}$ converging to a function $u$ in $L^{1}$. By a standard result on $\Gamma$-convergence, $u$ is a minimizer of $G_{\phi}$ and $G_{h}\left(u_{h}\right) \rightarrow G_{\phi}(u)$. In other terms, the minimizers $u_{h_{n}}$ in $V_{h_{n}}$ approximate the minimizer $u$ with respect to the $L^{1}$-topology while the energies $G_{h_{n}}\left(u_{h_{n}}\right)$ converge to $G_{\phi}(u)$.

If $f$ is concave, as is the case in most applications, by $11 f^{\prime}$ is non-increasing and $0 \leqslant f^{\prime}(t) \leqslant 1$. Therefore, the damage variable $d=1-f^{\prime}$ will be non-decreasing and such that

$$
\lim _{t \rightarrow 0^{+}} d(t)=0, \quad \lim _{t \rightarrow+\infty} d(t)=1 .
$$

Thus we can write

$$
\frac{\partial W_{h}}{\partial \varepsilon}=f^{\prime}\left(h W^{e}(\varepsilon)\right) \frac{\partial W^{e}}{\partial \varepsilon}=\left(1-d\left(h W^{e}(\varepsilon)\right)\right) \sigma,
$$


which is a standard form for a damage constitutive law. Usual choices for $f$ are

$$
f(t)=1-e^{-t}, \quad f(t)=t \wedge 1= \begin{cases}t & \text { for } t<1, \\ 1 & \text { otherwise }\end{cases}
$$

The first, being smooth, is more suitable for numerical computations and resembles the behaviour of quasi-brittle materials, such as concrete. On the other hand, the second choice better reveals the underlying idea of this approach and introduces a sharp elasto-fracture transition which is typical of brittle materials. In this case the energy density $W_{h}$ can be written as

$$
W_{h}\left(\varepsilon\left(u_{h}\right)\right)= \begin{cases}W^{e}\left(\varepsilon\left(u_{h}\right)\right) & \text { if } W^{e}\left(\varepsilon\left(u_{h}\right)\right)<1 / h, \\ 1 / h & \text { if } W^{e}\left(\varepsilon\left(u_{h}\right)\right) \geqslant 1 / h .\end{cases}
$$

The first regime represents clearly the elastic behaviour. The second accounts for fractures, indeed in this case the local energy is simply $|T| / h=h / 4$. This quantity represents the (anisotropic) length of a crack "embedded" in the element $T$ and resembles a similar argument of [25]. Note that this is the only energy "stored" in $T$ and thus, once a fracture is created, the element is considered traction free, in agreement with the equilibrium (Euler-Lagrange) equations. Finally, we remark that the fracture criterion depends on the element size $h$ and scales like $1 / h$. This order of convergence is in accordance with the behaviour of the strain in the vicinity of the crack tip. Indeed, it is well known that in the continuous model the strain field $u$ has a singularity of the form $\sqrt{r}$ (in the standard system $(r, \theta)$ of polar coordinates centred at the crack tip). In the discrete setting, considering the Lagrange interpolation $u_{h}$ of $u$, the elastic energy density $W^{e}$ will be just of the form $1 / h$.

Finally, let us remark that the isotropic fracture energy appearing in Griffith's model can be written as

$$
\gamma|J(u)|=\gamma \int_{J(u)}|\nu| \mathrm{d} s=\gamma \int_{J(u)} \mathrm{d} s,
$$

where $|v|$ denotes the usual Euclidean norm. It is clear that $\phi$ measures the distortion from the isotropic case due to the triangulation $\boldsymbol{T}_{h}$.

\section{Definition and properties of the mesh bias}

The anisotropy function $\phi$, appearing in 3 , can be defined in several equivalent ways. Let $\widehat{T}$ be a triangle of $\boldsymbol{T}_{h}$ for $h=1$, i.e. with diameter equal to one, and let $v$ be a vector in $\mathbb{R}^{2}$. For every $\xi \in \mathbb{S}^{1}$ (the unit circle) let $\tau_{\xi}$ be the height of $\widehat{T}$ in direction $\xi$ (see Figure 3 . Then we can define

$$
\phi(v)=\sup \left\{\tau_{\xi}\langle v, \xi\rangle: \xi \in \mathbb{S}^{1}\right\}
$$

As a matter of fact, $\phi(v)$ can be written more easily considering only a finite number of vectors $\xi$. Indeed, $\xi \mapsto \tau_{\xi} \xi$ is a one-to-one mapping of $\mathbb{S}^{1}$ into $\partial H$, the boundary of the hexagon $H$ obtained by taking the union of the triangles $\widehat{T}$ with a common vertex at the origin. Then we can write

$$
\phi(v)=\sup \{\langle v, y\rangle: y \in \partial H\}=\sup \{|\langle v, y\rangle|: y \in \partial H\},
$$

which is clearly equivalent to (5). As $H$ is a convex set, we get $\phi(v)=\sup \left\{\left\langle v, y_{i}\right\rangle: y_{i}\right.$ are the vertices of $H$ \}. Writing $y_{i}=\tau_{i} \xi_{i}$ we get

$$
\phi(v)=\sup \left\{\tau_{i}\left|\left\langle v, \xi_{i}\right\rangle\right|: i=1, \ldots, 3\right\}
$$


where

$$
\begin{array}{lll}
\xi_{1}=(1,0), & \xi_{2}=(\sqrt{2} / 2, \sqrt{2} / 2), & \xi_{3}=(0,1), \\
\tau_{1}=\sqrt{2} / 2, & \tau_{2}=1, & \tau_{3}=\sqrt{2} / 2 .
\end{array}
$$

It follows that $\phi$ is a norm in $\mathbb{R}^{2}$. Its unit ball $\{\phi(v)=1\}$ is represented in Figure 2 and shows clearly a strong dependence on the orientation. Finally, from 6 we can regard $\phi(v)$ (for $\left.v \in \mathbb{S}^{1}\right)$ as the one-dimensional measure of $\widehat{T}_{v}$, the projection of $\widehat{T}$ on the subspace $\langle v\rangle$.

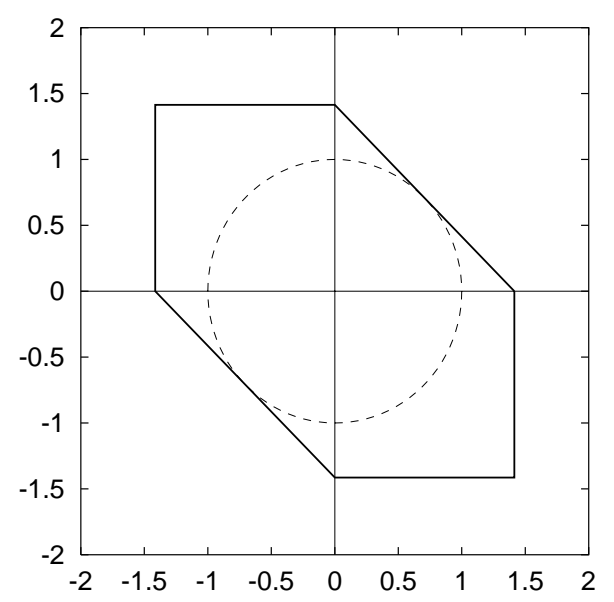

FIG. 2. The anisotropic unit ball $\{\phi(v)=1\}$ compared with the unit circle $\{|v|=1\}$.

REMARK 3.1 Whenever the topology of the mesh is similar to that of Figure 1 it is possible to compute the associated anisotropy function $\phi$. More precisely, assume that the triangulation has the following periodicity: the union of the elements with a common vertex is a hexagon $H$ which, up to translations, is independent of the vertex itself. Under this hypothesis $\phi$ can be computed as in (6) or (7). For instance, considering a mesh of equilateral triangles, the set $H$ will be a regular hexagon and then the unit ball of the anisotropy $\phi$ will be a regular hexagon as well (see [22]).

The definition of $\phi$ given in (5) is designed mainly for the proof of the $\Gamma$-liminf inequality. For the $\Gamma$-limsup inequality we will use instead the following property.

LEMMA 3.2 Let $J$ be a segment in $\mathbb{R}^{2}$ with normal $v$. Let $h_{n}$ be an infinitesimal sequence. Assume that for every $h_{n}$ the set $J$ does not contain any vertex of the triangulation $\boldsymbol{T}_{h_{n}}$ and define the covering $J_{h_{n}}$ as the union of the triangles $T$ of $\boldsymbol{T}_{h_{n}}$ which intersect $J$ (see Figure 3 ). Then

$$
\limsup _{n \rightarrow+\infty} \frac{\left|J_{h_{n}}\right|}{h_{n}}=\phi(v) \mathcal{H}^{1}(J) \text {. }
$$

Proof. Let $\zeta \in \mathbb{S}^{1}$ be such that $\phi(v)=\tau_{\zeta}\langle\zeta, v\rangle$ (see Figure 3). For $z \in J$ let $J_{h}^{z}$ be the onedimensional section $\left\{y \in J_{h}: y=z+s \zeta\right.$ for $\left.s \in \mathbb{R}\right\}$. Since $\zeta$ is aligned with the lattice $(h / \sqrt{2}) \mathbb{Z}^{2}$ it is not difficult to check that the measure of $J_{h}^{z}$ does not depend on $z$ and is equal to $\tau_{\zeta} h$. Thus we 

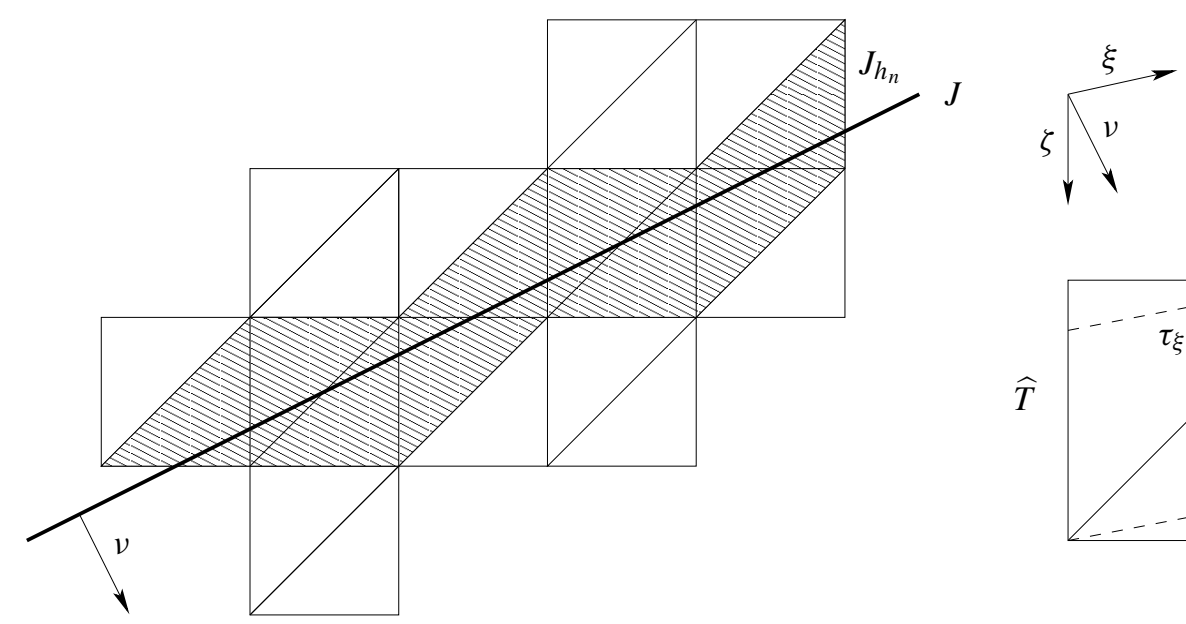

FIG. 3. The covering $J_{h_{n}}$ of a segment $J$ and the height $\tau_{\xi}$.

have $\left|J_{h_{n}}\right|=\mathcal{H}^{1}(J) \tau_{\zeta} h_{n}\langle\zeta, v\rangle+O\left(h_{n}^{2}\right)$, where $O\left(h_{n}^{2}\right)$ takes into account the behaviour close to the endpoints of $J$. As $h_{n} \rightarrow 0$ we get (8).

An alternative proof can be found in [22, Lemma 3.3].

Now, let us consider the example represented in Figure 4 A fracture set $J(u)$ is plotted with two triangulations $\boldsymbol{T}_{h}$ having different sizes. The elements covering the fracture give the smeared representation of $J(u)$. We can clearly see that such coverings form two neighbourhoods of $J(u)$ which are not uniform and depend strongly on the orientation of the curve (i.e. on its normal $v)$. The difference is really evident comparing the behaviour close to the endpoints of $J(u)$. As shown in the right picture, this phenomenon does not change when the size becomes smaller. In particular it will not disappear as $h \rightarrow 0$ and in the limit it will generate the anisotropic density $\phi$.

Finally, we remark that in the case of a general family of regular triangulations, it is not possible to find a $\Gamma$-limit and thus to define an anisotropy function. Indeed, the usual regularity property of the meshes is not enough: it is invariant under rigid motions and thus it cannot give any information on the orientation of the elements, which by contrast is fundamental for the definition of the coverings $J_{h}$ and then in the computation of (8). Moreover, it is well known that unstructured triangulations do not reduce these anisotropy effects. Better results are obtained when the coverings $J_{h}$ are close to a uniform (tubular) neighbourhood of $J$. This is the case when we employ adaptive triangulations (e.g. [6] for Mumford-Shah and [7] for Griffith) or non-local functionals (e.g. [9] for Mumford-Shah and [24] for Griffith) of the form

$$
G_{h}\left(u_{h}\right)=\int_{\Omega} f_{h}\left(W^{e}\left(\varepsilon\left(u_{h}\right)\right) * \rho_{h}\right) .
$$

The non-local operator, with weight $\rho_{h}$ and radius $r_{h}$, is denoted by $W^{e}\left(\varepsilon\left(u_{h}\right)\right) * \rho_{h}$ (a convolution with kernel $\rho_{h}$ ) while the mesh size $h$ is assumed to be sufficiently smaller than $r_{h}$. Often this radius is called "internal length" and is considered a physical parameter depending on the properties of the material at the meso scale. It seems interesting to understand the behaviour when the internal length is very small, namely when $r_{h} \rightarrow 0$ : from the mechanical point of view we will expect 


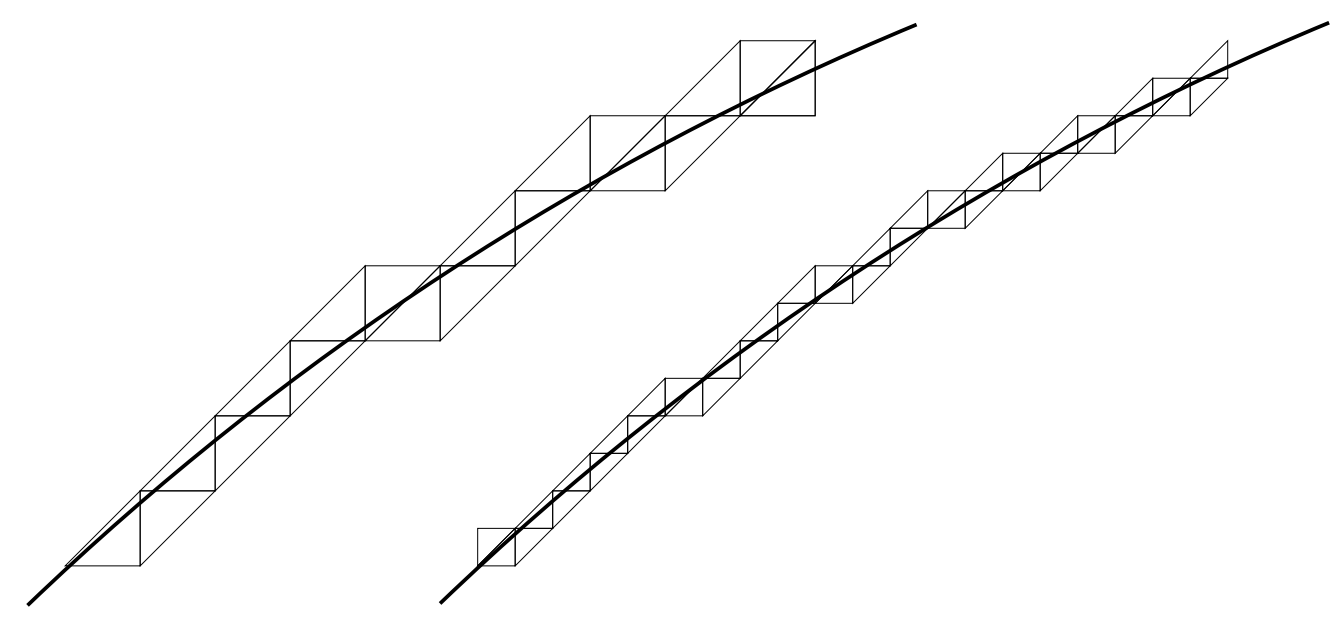

FIG. 4. A fracture set and its smeared representation for two mesh sizes.

a transition between a quasi-brittle and a brittle material, while from the mathematical point of view we will be able to estimate the mesh bias. More precisely, let us assume that $\rho_{h}$ is obtained by $\rho_{h}(z)=\rho(z / h) / h$. Now, let $f_{h}(t)=f\left(r_{h} t\right) / r_{h}$. Note that here the scaling factor is $r_{h}$. It is reasonable to expect that for $r_{h} \rightarrow 0$ the energy $G_{h}$ will converge again to a functional $G_{\phi}$ like (2) where the anisotropy $\phi$ will be closer to the Euclidean norm. When $h=o\left(r_{h}\right)$ this result has been proved in [14] for the Mumford-Shah functional and, by similar arguments, follows from [24] for Griffith.

\section{The $\Gamma$-limsup inequality}

For our type of functional the $\Gamma$-limsup inequality is almost straightforward and based on standard arguments. For completeness we give a short sketch which touches the main points of the proof, referring to [13] or [22] for the details in a similar case. Let us consider a sequence $h_{n} \rightarrow 0$ and the discrete functionals

$$
G_{n}(u)= \begin{cases}\int_{\Omega} W_{n}(\varepsilon(u)) \mathrm{d} x, & u_{n} \in V_{n}, \\ +\infty, & u_{n} \in L^{1} \backslash V_{n} .\end{cases}
$$

(For simplicity in the notation the subscript $h_{n}$ is replaced by $n$.)

Thanks to a recent density result [12] (see also Proposition A.3] it is sufficient to take into account a class of very regular functions of bounded deformation, namely those functions $u$ such that $J(u)$ is the union of the disjoint segments $J^{i}$ (for $i=1, \ldots, m$ ) and such that $u$ belongs to the Sobolev space $W^{k, \infty}(\Omega \backslash J(u))$ (for some $k$ arbitrarily large). By a simple translation argument, it is not restrictive to assume that $J(u)$ does not contain any vertex of the triangulations $\boldsymbol{T}_{n}$. In this way we can define $u_{n}$ as the Lagrange interpolation of $u$ in the space $V_{n}$. We denote by $J_{n}^{i}$ the coverings of $J^{i}$ as defined in the previous section and let $J_{n}$ be their union. Clearly we can write that

$$
\limsup _{n \rightarrow+\infty} G_{n}\left(u_{n}\right) \leqslant \limsup _{n \rightarrow+\infty} \int_{\Omega \backslash J_{n}} W_{n}\left(\varepsilon\left(u_{n}\right)\right) \mathrm{d} x+\limsup _{n \rightarrow+\infty} \int_{J_{n}} W_{n}\left(\varepsilon\left(u_{n}\right)\right) \mathrm{d} x .
$$


As $\left|J_{n}\right| \rightarrow 0$, by the regularity of $u$ and classical results on finite element interpolation, the first limit gives the bulk energy, i.e.

$$
\limsup _{n \rightarrow+\infty} \int_{\Omega \backslash J_{n}} W_{n}\left(\varepsilon\left(u_{n}\right)\right) \mathrm{d} x \leqslant \int_{\Omega \backslash J(u)} W^{e}\left(\varepsilon\left(u_{n}\right)\right) \mathrm{d} x .
$$

The second limit will give the fracture energy. Indeed, as $W_{n}\left(\varepsilon\left(u_{n}\right)\right) \leqslant \gamma$, by Lemma 3.2 we can write

$$
\begin{aligned}
\limsup _{n \rightarrow+\infty} \int_{J_{n}} W_{n}\left(\varepsilon\left(u_{n}\right)\right) \mathrm{d} x & \leqslant \sum_{i=1}^{m} \limsup _{n \rightarrow+\infty} \int_{J_{n}^{i}} W_{n}\left(\varepsilon\left(u_{n}\right)\right) \mathrm{d} x \leqslant \gamma \sum_{i=1}^{m} \limsup _{n \rightarrow+\infty} \frac{\left|J_{n}^{i}\right|}{h_{n}} \\
& \leqslant \gamma \sum_{i=1}^{m} \phi(\nu) \mathcal{H}^{1}\left(J^{i}\right)=\gamma \int_{J(u)} \phi(v) \mathrm{d} s .
\end{aligned}
$$

It follows easily that $\lim \sup _{n \rightarrow+\infty} G_{n}\left(u_{n}\right) \leqslant G_{\phi}(u)$.

\section{The $\Gamma$-liminf inequality}

The proof of the $\Gamma$-liminf inequality is based on a measure-theoretic argument (Lemma A.4 which allows considering separately the estimate for the bulk and surface energy. As it is not restrictive, we will consider again the sequence of functionals $G_{n}$ defined in the previous section. Moreover, for every open subset $A$ of $\Omega$, the localized functionals will be defined as

$$
\begin{aligned}
G_{n}(u, A) & = \begin{cases}\int_{A} W_{n}(\varepsilon(u)) \mathrm{d} x, & u \in V_{n}, \\
+\infty, & u \in L^{1} \backslash V_{n},\end{cases} \\
G(u, A) & = \begin{cases}\int_{A \backslash J(u)} W^{e}(\varepsilon(u)) \mathrm{d} x+\int_{J(u) \cap A} \phi(v) \mathrm{d} s, & u \in S B D^{2}, \\
+\infty, & u \in L^{1} \backslash S B D^{2} .\end{cases}
\end{aligned}
$$

\subsection{Estimate for the bulk energy and compactness}

For $0<\delta<1$ let $\delta^{\prime}>0$ be such that $f(t) \geqslant(1-\delta) t \wedge \delta^{\prime}$. For $A \subset \Omega$ and $\eta>0$ let $A_{\eta}=\{x \in A$ : $d(x, \partial A)>\eta$ \}. First we will show that (if $n$ is sufficiently large) for every $u \in V_{n}$ there exists $v \in S B D$ (depending on $\delta$ ) such that $\|v-u\|_{L^{1}} \leqslant\|u\|_{L^{\infty}} h_{n} G_{n}(u, A) / \delta^{\prime}$ and

$$
(1-\delta) \int_{A_{\eta} \backslash J(v)} W^{e}(\varepsilon(v)) \mathrm{d} x+c \delta^{\prime} \mathcal{H}^{1}\left(J(v) \cap A_{\eta}\right) \leqslant G_{n}\left(u_{n}, A\right),
$$

where the parameter $c>0$ depends only on the geometry of the mesh. From this estimate we will prove both compactness and the lower inequality for the bulk energy.

The proof of [11 follows closely [13] and therefore we just give a short sketch. Let $f^{\delta}(t)=$ $(1-\delta) t \wedge \delta^{\prime}$ and $f_{n}^{\delta}(t)=f^{\delta}\left(h_{n} t\right) / h_{n}=(1-\delta) t \wedge\left(\delta^{\prime} / h_{n}\right)$. Then

$$
W_{n}(\varepsilon(u))=f_{n}\left(W^{e}(\varepsilon(u))\right) \geqslant f_{n}^{\delta}\left(W^{e}(\varepsilon(u))\right) .
$$


Using the function $f_{n}^{\delta}$, we can clearly distinguish the elastic and fracture regimes. Indeed,

$$
f_{n}^{\delta}\left(W^{e}(\varepsilon(u))\right)= \begin{cases}(1-\delta) W^{e}(\varepsilon(u)) & \text { if } h_{n}(1-\delta) W^{e}(\varepsilon(u))<\delta^{\prime} \\ \delta^{\prime} / h_{n} & \text { if } h_{n}(1-\delta) W^{e}(\varepsilon(u)) \geqslant \delta^{\prime} .\end{cases}
$$

According to this behaviour we introduce the (relatively closed) set $A_{n}^{\sharp}$ which represents the smeared fracture and is obtained by the intersection of $A$ with all the triangles $T$ where $h_{n}(1-\delta) W^{e}(\varepsilon(u))$ $\geqslant \delta^{\prime}$. Finally, we define $A_{n}^{\mathrm{b}}=A \backslash A_{n}^{\sharp}$ and using this notation we obtain the lower bound

$$
G_{n}(u, A) \geqslant(1-\delta) \int_{A_{n}^{\mathrm{b}} \backslash J(u)} W^{e}(\varepsilon(u)) \mathrm{d} x+\delta^{\prime}\left|A_{n}^{\sharp}\right| / h_{n} .
$$

Let the function $v$ be defined as

$$
v= \begin{cases}u & \text { in } A_{n}^{b}, \\ 0 & \text { elsewhere. }\end{cases}
$$

Obviously $v \in S B D^{2}$ and $J(v) \subset \partial A_{n}^{\sharp}$. Since $v=u$ in $A_{n}^{b}$, from 12 we get easily $\|v-u\|_{L^{1}} \leqslant$ $\|u\|_{L^{\infty}} h_{n} G_{n}(u, A) / \delta^{\prime}$, while inequality 111 will be obtained by estimating the $\mathcal{H}^{1}$-measure of $J(v)$ in terms of $\left|A_{n}^{\sharp}\right| / h_{n}$. Clearly $J(v)$ is covered by the boundaries of the elements $T$ which form $A_{n}^{\sharp}$. For a suitable constant $c$ (independent of $h_{n}$ ) we can write $|T|=c h_{n} \mathcal{H}^{1}(\partial T)$ for every triangle $T$. Therefore we could get $(11)$ from $(12)$ if $|T \cap A|=c h_{n} \mathcal{H}^{1}(\partial T \cap A)$. Unfortunately, this bound is not always true: it may happen that an element $T$ crosses the boundary of $A$ in such a way that $|T \cap A|$ is less than $c h_{n} \mathcal{H}^{1}(\partial T \cap A)$. This small difficulty can be easily overcome by considering the estimate only in the set $A_{\eta}$ and choosing $n$ sufficiently large, in such a way that $h_{n}<\eta$.

Now we can prove the liminf inequality for the bulk energy. Let $u_{n} \in V_{n}$ converge to $u$ in $L^{1}(\Omega)$. For an open subset $A$ of $\Omega$, after passing to a subsequence (not relabelled) we can assume without loss of generality that

$$
\liminf _{n \rightarrow+\infty} G_{n}\left(u_{n}, A\right)=\lim _{n \rightarrow+\infty} G_{n}\left(u_{n}, A\right)<+\infty .
$$

Now, for $\delta>0$ let $v_{n} \in S B D$ satisfy (11). Since

$$
\left\|v_{n}-u_{n}\right\|_{L^{1}} \leqslant\left\|u_{n}\right\|_{L^{\infty}} h_{n} G_{n}\left(u_{n}, A\right) / \delta^{\prime}
$$

we get $v_{n} \rightarrow u$ in $L^{1}(\Omega)$. Then for every open set $A \subset \Omega$ by lower semicontinuity we obtain

$$
\begin{aligned}
(1-\delta) \int_{A_{\eta} \backslash J(u)} W^{e}(\varepsilon(u)) \mathrm{d} x & \leqslant \liminf _{n \rightarrow+\infty}(1-\delta) \int_{A_{\eta} \backslash J\left(v_{n}\right)} W^{e}\left(\varepsilon\left(v_{n}\right)\right) \mathrm{d} x \\
& \leqslant \liminf _{n \rightarrow+\infty} G_{n}\left(u_{n}, A\right) .
\end{aligned}
$$

Taking the supremum as $\eta \searrow 0$ we get the localized lower inequality for the bulk energy:

$$
\int_{A \backslash J(u)} W^{e}(\varepsilon(u)) \mathrm{d} x \leqslant \liminf _{n \rightarrow+\infty} G_{n}\left(u_{n}, A\right) .
$$


Finally, we can prove compactness. Let $u_{n} \in V_{n}$ be a sequence with bounded energy. For $\delta>0$ let $v_{n} \in S B D$ satisfying (11) for $A=\Omega$, namely

$$
(1-\delta) \int_{\Omega_{\eta} \backslash J\left(v_{n}\right)} W^{e}\left(\varepsilon\left(v_{n}\right)\right) \mathrm{d} x+c \delta^{\prime} \mathcal{H}^{1}\left(J\left(v_{n}\right) \cap \Omega_{\eta}\right) \leqslant G_{n}\left(u_{n}\right) \leqslant C .
$$

By [5] it follows that $v_{n}$ is precompact in $L^{1}\left(\Omega_{\eta}\right)$. Since $\left\|v_{n}-u_{n}\right\| \leqslant h_{n} C / \delta^{\prime}$ the sequence $u_{n}$ is precompact in $L^{1}\left(\Omega_{\eta}\right)$. As $\delta$ is arbitrarily small and $u_{n}$ is uniformly bounded in $L^{\infty}(\Omega)$ it follows easily that $u_{n}$ is precompact in $L^{1}(\Omega)$.

\subsection{Slicing estimate for the fracture energy}

Let us consider a sequence $u_{n} \in V_{n}$ converging to $u$ in $L^{1}(\Omega)$ and satisfying $(13)$. In this section we have to prove that

$$
\liminf _{n \rightarrow+\infty} G_{n}\left(u_{n}, A\right) \geqslant \gamma \int_{J(u) \cap A} \phi(v) \mathrm{d} s .
$$

The first step will be the following: for every vector of the form $\xi=\zeta /|\zeta|$, where $\zeta \in \mathbb{Z}^{2}$, we have

$$
\liminf _{n \rightarrow+\infty} G_{n}\left(u_{n}, A\right) \geqslant \gamma \int_{J \xi(u) \cap A} \eta_{\xi}|\langle\nu, \xi\rangle| \mathrm{d} s,
$$

for a suitable choice of $\eta_{\xi} \leqslant \tau_{\xi}$ to be specified later. As a matter of fact, it will be sufficient to show that

$$
\liminf _{n \rightarrow+\infty} \int_{A} f_{n}\left(\mu\left|D_{\xi} u_{n}^{\xi}\right|^{2}\right) \mathrm{d} x \geqslant \gamma \int_{J \xi(u) \cap A} \eta_{\xi}|\langle\nu, \xi\rangle| \mathrm{d} s
$$

Indeed, by Proposition A.2 we get

$$
W^{e}(\varepsilon(u)) \geqslant \mu|\varepsilon(u)|^{2} \geqslant \mu\|\varepsilon(u)\|^{2} \geqslant \mu|\langle\xi, \varepsilon(u) \xi\rangle|^{2}=\mu\left|D_{\xi} u^{\xi}\right|^{2}
$$

and then, $f_{n}$ being non-decreasing, we can write

$$
W_{n}(\varepsilon(u))=f_{n}\left(W^{e}(\varepsilon(u))\right) \geqslant f_{n}\left(\mu\left|D_{\xi} u^{\xi}\right|^{2}\right) .
$$

As anticipated, in this section we will use the slicing technique and thus we will need some more notation (see Figures 6 and 7 . For $\xi=\zeta /|\zeta|$ and $\zeta \in \mathbb{Z}^{2}$ let $\xi^{\prime} \in\left\{\xi_{i}\right\}$ be such that $\left|\left\langle\xi, \xi^{\prime}\right\rangle\right|=$ $\min _{i}\left|\left\langle\xi, \xi_{i}\right\rangle\right|$. (The vector $\xi^{\prime}$ will replace $\xi^{\perp}$ in the slicing estimates.) Let us denote by $Y=\langle\xi\rangle$ and by $Z=\left\langle\xi^{\prime}\right\rangle$ the subspaces generated by $\xi$ and $\xi^{\prime}$. Now, considering a square $Q_{n}$ obtained as the union of two triangles $T_{n}$ of $\boldsymbol{T}_{n}$ (see Figure 5, we define $h_{n}^{\xi}$ and $b_{n}^{\xi}$ as the lengths of the sections of $Q_{n}$ in directions $\xi$ and $\xi^{\prime}$ respectively. Then $2\left|T_{n}\right|=h_{n}^{\xi} b_{n}^{\xi} c_{\xi}$ where $c_{\xi}=\left|\left\langle\xi^{\prime}, \xi^{\perp}\right\rangle\right|$. Note that the intersection of the set of knots $\left(h_{n} / \sqrt{2}\right) \mathbb{Z}^{2}$ and the subspace $Z$ will be a uniform grid of the form $b_{n}^{\xi} \mathbb{Z}$.

At this point, for the sake of clarity, it is better to consider separately the easier case $\xi=\xi_{i}$ (for $i=1, \ldots, 3)$ and the general case $\xi \neq \xi_{i}$. 

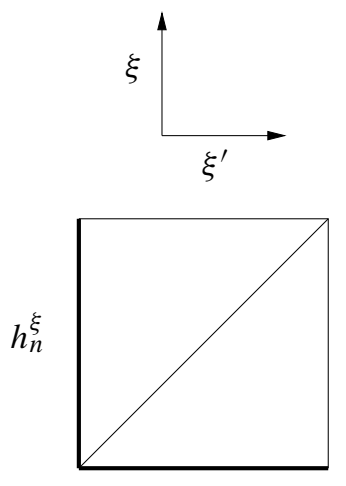

$b_{n}^{\xi}$
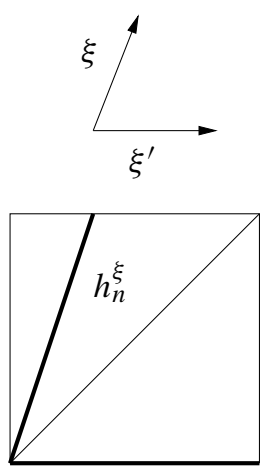

$b_{n}^{\xi}$
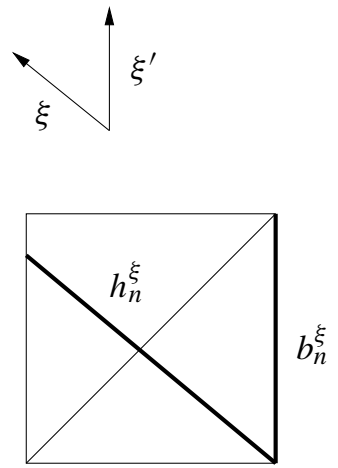

FIG. 5. Some examples for the definition of $h_{n}^{\xi}$ and $b_{n}^{\xi}$.

5.2.1 Slicing estimate for $\xi=\xi_{i}$. Let $A$ be an open subset of $\Omega$. For $z \in Z$ let $A^{\xi, z}$ be the section $\{x \in A: x=z+t \xi$ for $t \in \mathbb{R}\}$. For $z \in b_{n}^{\xi} \mathbb{Z}$ we denote by $\boldsymbol{A}_{n}^{\xi, z}$ the closed stripe obtained as the union of the (closed) squares $Q_{n}^{\xi, z}$ which are contained in $A$ and which cover the section $A^{\xi, z}$ as represented in Figure 6 As the sets $\boldsymbol{A}_{n}^{\xi, z}$ are pairwise disjoint (up to a set of measure zero) we can write

$$
\int_{A} f_{n}\left(\mu\left|D_{\xi} u_{n}^{\xi}\right|^{2}\right) \mathrm{d} x \geqslant \sum_{z \in b_{n}^{\xi} \mathbb{Z}} \int_{A_{n}^{\xi, z}} f_{n}\left(\mu\left|D_{\xi} u_{n}^{\xi}\right|^{2}\right) \mathrm{d} x .
$$

For every stripe $\boldsymbol{A}_{n}^{\xi, z}$ let us denote by $\partial_{L} \boldsymbol{A}_{n}^{\xi, z}$ and $\partial_{R} \boldsymbol{A}_{n}^{\xi, z}$ the left and right sides of $\boldsymbol{A}_{n}^{\xi, z}$, i.e.

$$
\partial_{L} \boldsymbol{A}_{n}^{\xi, z}=\left\{x \in \boldsymbol{A}_{n}^{\xi, z}: x=z+s \xi\right\}, \quad \partial_{R} \boldsymbol{A}_{n}^{\xi, z}=\left\{x \in \boldsymbol{A}_{n}^{\xi, z}: x=\left(z+b_{n}^{\xi}\right)+s \xi\right\} .
$$

Let $l_{n}^{\xi, z}$ and $r_{n}^{\xi, z}$ denote the restrictions of $u_{n}^{\xi}$ respectively to $\partial_{L} \boldsymbol{A}_{n}^{\xi, z}$ and $\partial_{R} \boldsymbol{A}_{n}^{\xi, z}$. By abuse of notation, we will consider $l_{n}^{\xi, z}$ and $r_{n}^{\xi, z}$ to be defined also on a one-dimensional lattice of the form $i h_{n}^{\xi}$ (for $i=0, \ldots, k_{n}$ and $k_{n}$ depending on $z$ ) which represents the knots lying on the slices $\partial_{L, R} \boldsymbol{A}_{n}^{\xi, z}$. Then, whenever possible, we will drop the dependence on $\xi$ and $z$ and we will denote by $l_{n}^{i}$ the value $l_{n}^{\xi, z}\left(i h_{n}^{\xi}\right)$.

Clearly on every stripe $A_{n}^{\xi, z}$ we can write $D_{\xi} u_{n}^{\xi}$ in terms of $l_{n}^{\xi, z}$ and $r_{n}^{\xi, z}$. As $\xi$ is aligned with the edges of the elements, for every triangle $T_{n}$ we have either

$$
D_{\xi} u_{n}^{\xi}=\frac{l_{n}^{i+1}-l_{n}^{i}}{h_{n}^{\xi}}=D^{i} l_{n} \quad \text { or } \quad D_{\xi} u_{n}^{\xi}=\frac{r_{n}^{i+1}-r_{n}^{i}}{h_{n}^{\xi}}=D^{i} r_{n} .
$$

Thus

$$
\begin{aligned}
\int_{\boldsymbol{A}_{n}^{\xi, z}} f_{n}\left(\mu\left|D_{\xi} u_{n}^{\xi}\right|^{2}\right) \mathrm{d} x & =\sum_{i=0}^{k_{n}-1}\left(|T| f_{n}\left(\mu\left|D^{i} l_{n}\right|^{2}\right)+|T| f_{n}\left(\mu\left|D^{i} r_{n}\right|^{2}\right)\right) \\
& =\sum_{i=0}^{k_{n}-1}\left(\left(\frac{1}{2} b_{n}^{\xi} c_{\xi}\right) h_{n}^{\xi} f_{n}\left(\mu\left|D^{i} l_{n}\right|^{2}\right)+\left(\frac{1}{2} b_{n}^{\xi} c_{\xi}\right) h_{n}^{\xi} f_{n}\left(\mu\left|D^{i} r_{n}\right|^{2}\right)\right) .
\end{aligned}
$$




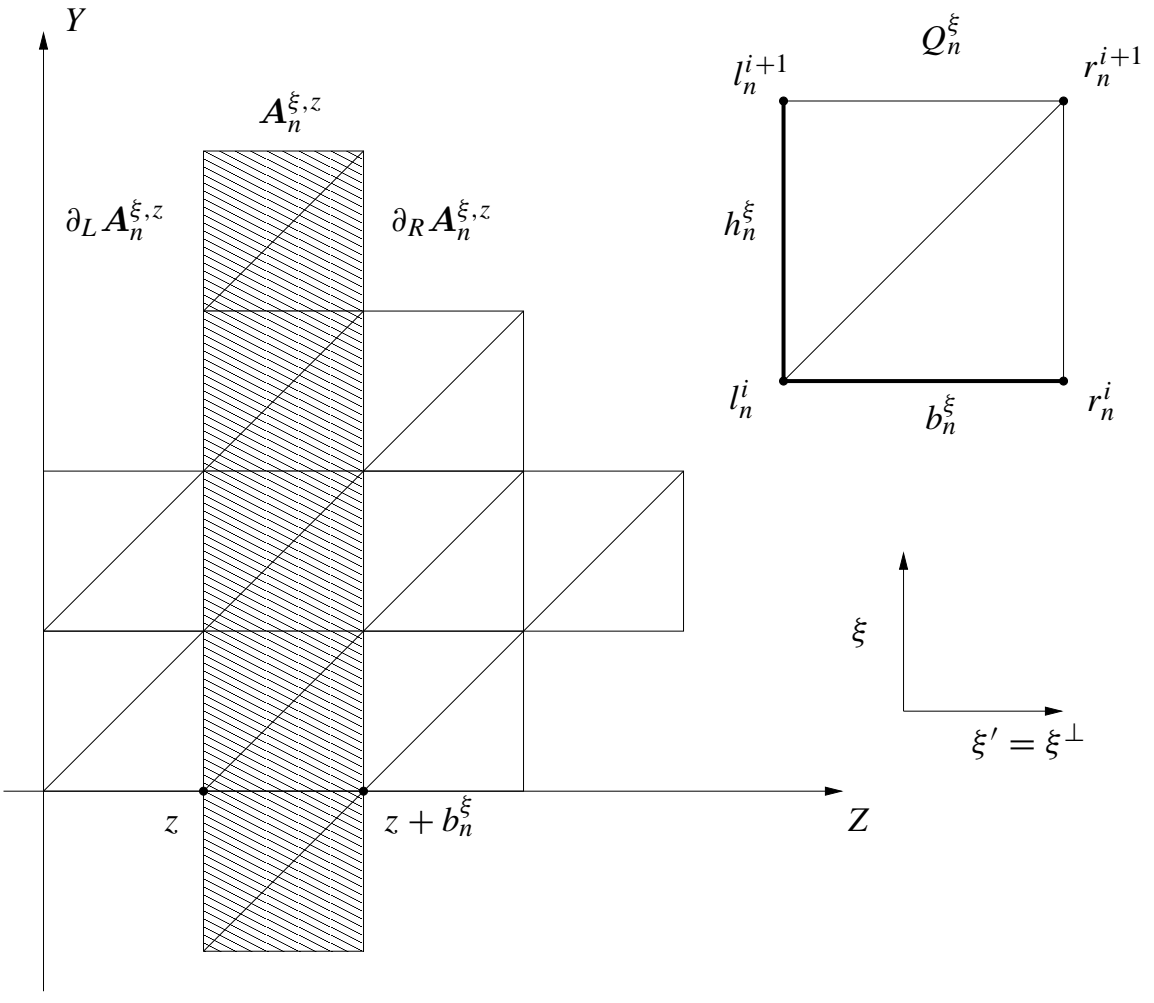

FIG. 6. Representation of the slicing for $\xi=\xi_{1} ; l_{n}^{i}$ denotes $l_{n}^{\xi, z}\left(i h_{n}^{\xi}\right)$ etc.

For $\eta_{\xi}=h_{n}^{\xi} / h_{n}$ and $\mu_{\xi}=\mu / \eta_{\xi}$, we define the one-dimensional functional

$$
F_{n}\left(l_{n}\right)=\sum_{i=0}^{k_{n}-1} h_{n}^{\xi} f_{n}\left(\mu\left|D^{i} l_{n}\right|^{2}\right)=\eta_{\xi} h_{n}^{\xi} \sum_{i=0}^{k_{n}-1} \frac{1}{h_{n}^{\xi}} f\left(h_{n}^{\xi} \mu_{\xi}\left|D^{i} l_{n}\right|^{2}\right) .
$$

Then we can write

$$
\int_{\boldsymbol{A}_{n}^{\xi, z}} f_{n}\left(\mu\left|D_{\xi} u_{n}^{\xi}\right|^{2}\right) \mathrm{d} x \geqslant\left(\frac{1}{2} b_{n}^{\xi} c_{\xi}\right) F_{n}\left(l_{n}\right)+\left(\frac{1}{2} b_{n}^{\xi} c_{\xi}\right) F_{n}\left(r_{n}\right)
$$

Let $l_{n}^{\xi}$ and $r_{n}^{\xi}$ be the (right and left) extensions of $l_{n}^{\xi, z}$ and $r_{n}^{\xi, z}$ to $A_{n}^{\xi, z}$ (for every $z \in b_{n}^{\xi} \mathbb{Z}$ ) defined by $l_{n}^{\xi}\left(x+t \xi^{\prime}\right)=l_{n}^{\xi, z}(x)$ for $x \in \partial_{L} A_{n}^{\xi, z}$ and $r_{n}^{\xi}\left(x-t \xi^{\prime}\right)=r_{n}^{\xi, z}(x)$ for $x \in \partial_{R} A_{n}^{\xi, z}$. If $x \notin A_{n}^{\xi, z}$ for any $z \in b_{n}^{\xi} \mathbb{Z}$ then we set $l_{n}^{\xi}=r_{n}^{\xi}=0$. Note that by definition $l_{n}^{\xi}$ and $r_{n}^{\xi}$ are piecewise affine on the squares $Q_{n}^{\xi, z}$ and are possibly discontinuous along $\partial_{L, R} A_{n}^{\xi, z}$. Moreover we have the following property.

LEMMA 5.1 The sequences $l_{n}^{\xi}$ and $r_{n}^{\xi}$ converge to $u^{\xi}$ in $L^{1}(A)$.

Proof. Let $m_{n}$ and $M_{n}$ be piecewise constant functions on the squares $Q_{n}^{\xi}$ defined by $m_{n}=$ $\min _{Q_{n}^{\xi}} u_{n}^{\xi}$ and $M_{n}=\max _{Q_{n}^{\xi}} u_{n}^{\xi}$, for every $Q_{n}^{\xi}$ (note that $u_{n}^{\xi}$ takes scalar values). Let $k \in \mathbb{N}$, and 
for $i, j \in \mathbb{N}$ with $|i| \leqslant k$ and $|j| \leqslant k$ let us introduce the function $w_{n}^{i, j}(x)=u_{n}^{\xi}\left(x+y_{i} \xi+z_{j} \xi^{\prime}\right)$ where $y_{i}=(i / k) h_{n}^{\xi}$ and $z_{j}=(i / k) b_{n}^{\xi}$. Finally, we consider the functions $M_{n}^{k}$ and $m_{n}^{k}$ given by

$$
M_{n}^{k}(x)=\max _{i, j}\left\{w_{n}^{i, j}(x)\right\}, \quad m_{n}^{k}(x)=\min _{i, j}\left\{w_{n}^{i, j}(x)\right\} .
$$

Clearly $w_{n}^{i, j} \rightarrow u^{\xi}$ for every $i, j$ and so $M_{n}^{k}$ and $m_{n}^{k}$ converge to $u^{\xi}$ as well. For $\eta>0$ let $A_{\eta}=$ $\{x \in A: d(x, \partial A)>\eta\}$. As $u_{n}^{\xi}$ is piecewise affine and uniformly bounded in $L^{\infty}$ it follows that for every $\delta>0$ there exists $k$, sufficiently large, such that for every $x \in A_{\eta}$ we have

$$
M_{n}^{k}(x) \geqslant M_{n}(x)-\delta, \quad m_{n}^{k}(x) \leqslant m_{n}(x)+\delta .
$$

Then for $n$ sufficiently large we can write $\left\|M_{n}^{k}-M_{n}\right\|_{L^{1}\left(A_{\eta}\right)} \leqslant \delta\left|A_{\eta}\right|$ and similarly for $m_{n}^{k}$. Since $\delta$ is arbitrary, by a diagonal argument it follows that $M_{n}$ and $m_{n}$ converge to $u^{\xi}$ as well. In the set $A_{\eta}$ we have $m_{n} \leqslant l_{n}^{\xi} \leqslant M_{n}$; thus we get $l_{n}^{\xi} \rightarrow u^{\xi}$, and the same for $r_{n}^{\xi}$. For $\eta \searrow 0$ we deduce the convergence in $L^{1}(A)$.

For $w \in Z$ let us denote by $l_{n}^{\xi, w}$ the restriction of $l_{n}^{\xi}$ to the section $A^{\xi, w}$. By definition $l_{n}^{\xi, w}=l_{n}^{\xi, z}$ for every $w \in\left[z, z+b_{n}^{\xi}\right)$. Hence we can write

$$
b_{n}^{\xi} F_{n}\left(l_{n}^{\xi, z}\right)=\int_{z}^{z+b_{n}^{\xi}} F_{n}\left(l_{n}^{\xi, w}\right) \mathrm{d} w
$$

and similarly for $r_{n}^{\xi}$. Then from 16 and 18 , we get

$$
\begin{aligned}
\int_{A} f_{n}\left(\mu\left|D_{\xi} u_{n}^{\xi}\right|^{2}\right) \mathrm{d} x & \geqslant \sum_{z \in b_{n}^{\xi} \mathbb{Z}} \int_{A_{n}^{\xi, z}} f_{n}\left(\mu\left|D_{\xi} u_{n}^{\xi}\right|^{2}\right) \mathrm{d} x \geqslant \sum_{z \in b_{n}^{\xi} \mathbb{Z}}\left(\left(\frac{1}{2} b_{n}^{\xi} c_{\xi}\right) F_{n}\left(l_{n}^{\xi, z}\right)+\left(\frac{1}{2} b_{n}^{\xi} c_{\xi}\right) F_{n}\left(r_{n}^{\xi, z}\right)\right) \\
& \geqslant\left(\frac{1}{2} c_{\xi}\right) \int_{Z} F_{n}\left(l_{n}^{\xi, w}\right) \mathrm{d} w+\left(\frac{1}{2} c_{\xi}\right) \int_{Z} F_{n}\left(r_{n}^{\xi, w}\right) \mathrm{d} w .
\end{aligned}
$$

By Lemma 5.1. $l_{n}^{\xi}$ converges to $u^{\xi} \chi_{A}$ in $L^{1}\left(\mathbb{R}^{2}\right)$ and thus $l_{n}^{\xi, w}$ converges to $u^{\xi, w} \chi_{A}$ for $\mathcal{H}^{1}$-a.e. $w \in Z$. Hence we get (see for instance [10])

$$
\liminf _{n \rightarrow+\infty} F_{n}\left(l_{n}^{\xi, w}\right) \geqslant \gamma \tau_{\xi} \#\left(J\left(u^{\xi, w}\right) \cap A^{\xi, w}\right) .
$$

Clearly the same holds for $r_{n}^{\xi, w}$. From this inequality we conclude easily; indeed, by Fatou's lemma and Proposition A.2 we get

$$
\begin{aligned}
\liminf _{n \rightarrow+\infty} \int_{A} f_{n}\left(\mu\left|D_{\xi} u_{n}^{\xi}\right|^{2}\right) \mathrm{d} x & \geqslant \gamma c_{\xi} \int_{Z} \tau_{\xi} \#\left(J\left(u^{\xi, w}\right) \cap A^{\xi, w}\right) \mathrm{d} w \\
& =\gamma \int_{\langle\xi \perp} \tau_{\xi} \#\left(J\left(u^{\xi, w}\right) \cap A^{\xi, w}\right) \mathrm{d} w=\gamma \int_{J^{\xi}(u) \cap A} \tau_{\xi}|\langle\nu, \xi\rangle| \mathrm{d} s .
\end{aligned}
$$

REMARK 5.2 Note that it is not possible to use simply one-dimensional restriction of the functional $G_{n}$ as given by Fubini's theorem. Such a choice would result in a wrong estimate of the surface energy due to the fact that the corresponding one-dimensional functional would be defined on a non-uniform lattice. In some sense the triangulation introduces a sort of non-local effect at 
the element scale $h_{n}$ which makes it impossible to consider directly the one-dimensional sections. Indeed, $G_{n}$ has been restricted first to the "smeared" section $A_{n}^{\xi, z}$ for $z \in b_{n}^{\xi} \mathbb{Z}$ and then, through the functions $l_{n}^{\xi}$ and $r_{n}^{\xi}$, to the "real" section $A^{\xi, w}$ for $w \in Z$.

5.2.2 Slicing estimate for $\xi \neq \xi_{i}$. For $\xi \neq \xi_{i}$ (in a similar way as for $\xi=\xi_{i}$ ) we define for $z \in b_{n}^{\xi} \mathbb{Z}$ the stripe $\boldsymbol{A}_{n}^{\xi, z}$ obtained this time as the union of the quadrilaterals $Q_{n}^{\xi, z}$ which are contained in $A$ and which cover the section $A^{\xi, z}$, as represented in Figure 7 The sets $Q_{n}^{\xi, z}$ are always the union of two neighbouring triangles of $\boldsymbol{T}_{n}$ which can form either a square or a parallelogram. Clearly, we can write the analogue of $(16)$, i.e.

$$
\int_{A} f_{n}\left(\mu\left|D_{\xi} u_{n}^{\xi}\right|^{2}\right) \mathrm{d} x \geqslant \sum_{z \in b_{n}^{\xi} \mathbb{Z}} \int_{A_{n}^{\xi, z}} f_{n}\left(\mu\left|D_{\xi} u_{n}^{\xi}\right|^{2}\right) \mathrm{d} x .
$$

Now, the definition of a suitable section in direction $\xi$ is more delicate, as the lattice $\left(h_{n} / \sqrt{2}\right) \mathbb{Z}^{2}$ is not aligned with $\xi$. As a matter of fact, we need first to replace the mesh $\boldsymbol{T}_{n}$ with a mesh $\boldsymbol{T}_{n}^{\prime}$ having knots in the lattice $\left(\xi^{\prime} b_{n}^{\xi} \mathbb{Z}\right) \times\left(\xi h_{n}^{\xi} \mathbb{Z}\right)$. This passage is done by means of a local transform, i.e. mapping the quadrilaterals $Q_{n}^{\xi, z}$ to parallelograms $P_{n}^{\xi, z}$ (see Figure 7). In this way every knot $x_{n}$ of $\boldsymbol{T}_{n}$ is mapped to a knot $x_{n}^{\prime}$ of $\boldsymbol{T}_{n}^{\prime}$, and each stripe $\boldsymbol{A}_{n}^{\xi, z}$ is mapped into a stripe $\boldsymbol{B}_{n}^{\xi, z}$ for which we can define $\partial_{L} \boldsymbol{B}_{n}^{\xi, z}$ and $\partial_{R} \boldsymbol{B}_{n}^{\xi, z}$ as we did in the previous section (see Figure 7). Finally, preserving the values at corresponding knots, the functions $u_{n}^{\xi}$ (defined on the mesh $\boldsymbol{T}_{n}$ ) will be replaced by $v_{n}^{\xi}$ (defined on the mesh $\boldsymbol{T}_{n}^{\prime}$ ) given by $v_{n}^{\xi}\left(x_{n}^{\prime}\right)=u_{n}^{\xi}\left(x_{n}\right)$.

REMARK 5.3 We remark that this transform does not coincide with the linear mapping of $\left(h_{n} / \sqrt{2}\right) \mathbb{Z}^{2}$ into $\left(\xi^{\prime} b_{n}^{\xi} \mathbb{Z}\right) \times\left(\xi h_{n}^{\xi} \mathbb{Z}\right)$. Such a (simpler) choice would not be right for our purposes. Moreover, observe that the mesh $T_{n}^{\prime}$ is not periodic with respect to $h_{n}^{\xi} \xi$. As shown in Figure 7 the orientation of the elements inside the parallelograms $P_{n}^{\xi, z}$ is no longer uniform.

Now we can define $l_{n}^{\xi, z}$ and $r_{n}^{\xi, z}$ as the restrictions of $v_{n}^{\xi}$ respectively to $\partial_{L} \boldsymbol{B}_{n}^{\xi, z}$ and $\partial_{R} \boldsymbol{B}_{n}^{\xi, z}$. The knots of $\boldsymbol{T}_{n}^{\prime}$ lying on $\partial_{L, R} \boldsymbol{B}_{n}^{\xi, z}$ define a uniform lattice: for simplicity of notation, we will write it in the form $i h_{n}^{\xi}$ for $i=-k_{n}, \ldots, k_{n}$ ( $k_{n}$ depending on $z$ ). As in the previous section, whenever possible we will drop the dependence on $\xi$ and $z$ and write $l_{n}^{i}$ for $l_{n}^{\xi, z}\left(i h_{n}\right)$. Finally, we will denote again by $l_{n}^{\xi}$ and $r_{n}^{\xi}$ their (right and left) extensions. Note that $v_{n}^{\xi}$ converges to $u^{\xi}$ in $L^{1}(A)$ and thus by Lemma 5.1 the same holds for $l_{n}^{\xi}$ and $r_{n}^{\xi}$.

Going back to the energy, the next step is to write the directional derivative $D_{\xi} u_{n}^{\xi}$ in terms of $l_{n}^{\xi, z}$ and $r_{n}^{\xi, z}$. Unfortunately, in this case $D_{\xi} u_{n}^{\xi}$ has a more complicated form due to the fact that $\xi$ is not aligned with the mesh $\boldsymbol{T}_{n}$. As $u_{n}^{\xi}$ is linear we can write its derivative $D_{\xi} u_{n}^{\xi}$ as a difference quotient along the height $h_{n}^{\xi}$. Let $\theta=\left|\xi \cdot \xi^{\prime}\right|$ be the projection of $\xi$ on $\xi^{\prime}$. It is now necessary to distinguish two cases (see Figure 7): if $Q_{n}^{\xi}$ is a square we get in the left and right triangle respectively

$$
\begin{aligned}
h_{n}^{\xi} D_{\xi} u_{n}^{\xi} & =l_{n}^{i+1}-\left[(1-\theta) r_{n}^{i}+\theta l_{n}^{i}\right] \\
& =\left(l_{n}^{i+1}-l_{n}^{i}\right)-(1-\theta)\left(r_{n}^{i}-l_{n}^{i}\right)=h_{n}^{\xi} D_{L}^{i}\left(l_{n}, r_{n}\right), \\
h_{n}^{\xi} D_{\xi} u_{n}^{\xi} & =\left[(1-\theta) l_{n}^{i+1}+\theta r_{n}^{i+1}\right]-r_{n}^{i} \\
& =\left(r_{n}^{i+1}-r_{n}^{i}\right)-(1-\theta)\left(r_{n}^{i+1}-l_{n}^{i+1}\right)=h_{n}^{\xi} D_{R}^{i}\left(l_{n}, r_{n}\right) ;
\end{aligned}
$$


SMEARED CRACK APPROACH
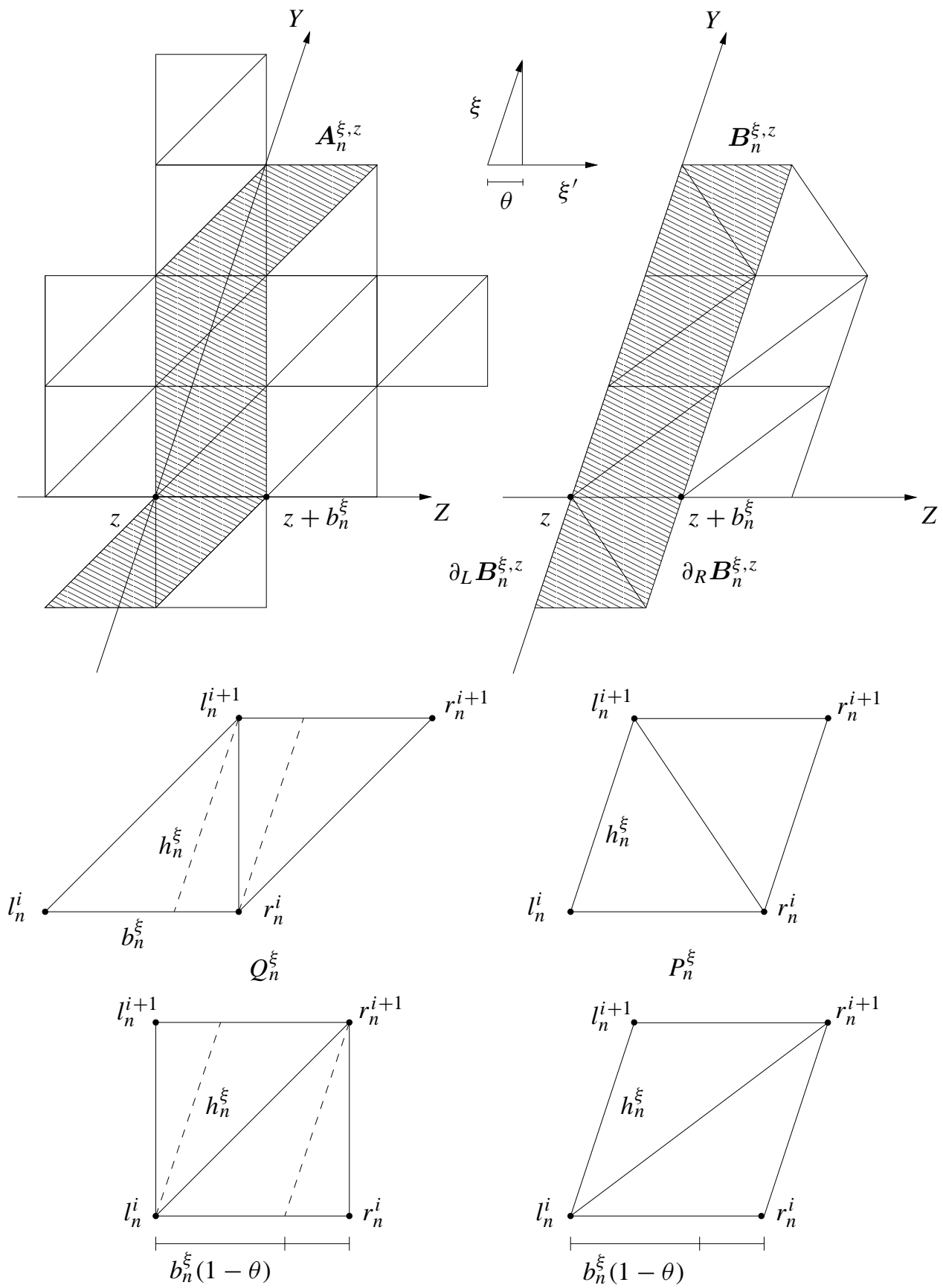

FIG. 7. Representation of the slicing for $\xi \neq \xi_{i}$. 
if $Q_{n}^{\xi}$ is not a square we get

$$
\begin{aligned}
h_{n}^{\xi} D_{\xi} u_{n}^{\xi} & =\left[(1-\theta) l_{n}^{i+1}+\theta r_{n}^{i+1}\right]-l_{n}^{i} \\
& =\left(l_{n}^{i+1}-l_{n}^{i}\right)+\theta\left(r_{n}^{i+1}-l_{n}^{i+1}\right)=h_{n}^{\xi} D_{L}^{i}\left(l_{n}, r_{n}\right), \\
h_{n}^{\xi} D_{\xi} u_{n}^{\xi} & =r_{n}^{i+1}-\left[(1-\theta) r_{n}^{i}+\theta l_{n}^{i}\right] \\
& =\left(r_{n}^{i+1}-r_{n}^{i}\right)+\theta\left(r_{n}^{i}-l_{n}^{i}\right)=h_{n}^{\xi} D_{R}^{i}\left(l_{n}, r_{n}\right) .
\end{aligned}
$$

Now, we will write the energy in the stripe $\boldsymbol{A}_{n}^{\xi, z}$ considering separately the left and right contributions, i.e.

$$
\begin{aligned}
\int_{\boldsymbol{A}_{n}^{\xi, z}} f_{n}\left(\mu\left|D_{\xi} u_{n}^{\xi}\right|^{2}\right) \mathrm{d} x & =\sum_{i=-k_{n}}^{k_{n}-1}\left(|T| f_{n}\left(\mu\left|D_{L}^{i}\left(l_{n}, r_{n}\right)\right|^{2}\right)+|T| f_{n}\left(\mu\left|D_{R}^{i}\left(l_{n}, r_{n}\right)\right|^{2}\right)\right) \\
& =\left(b_{n}^{\xi} c_{\xi}\right) \sum_{i=-k_{n}}^{k_{n}-1} \frac{1}{2} h_{n}^{\xi}\left(f_{n}\left(\mu\left|D_{L}^{i}\left(l_{n}, r_{n}\right)\right|^{2}\right)+f_{n}\left(\mu\left|D_{R}^{i}\left(l_{n}, r_{n}\right)\right|^{2}\right)\right) .
\end{aligned}
$$

This formula suggests the definition of the functional

$$
F_{n}\left(l_{n}, r_{n}\right):=\sum_{i=-k_{n}}^{k_{n}-1}\left(\frac{1}{2} h_{n}^{\xi} f_{n}\left(\mu\left|D_{L}^{i}\left(l_{n}, r_{n}\right)\right|^{2}\right)+\frac{1}{2} h_{n}^{\xi} f_{n}\left(\mu\left|D_{R}^{i}\left(l_{n}, r_{n}\right)\right|^{2}\right)\right) .
$$

Again, for $w \in Z$ we denote by $l_{n}^{\xi, w}$ the restriction of $l_{n}^{\xi}$ to the section $A^{\xi, w}$. As $l_{n}^{\xi, w}=l_{n}^{\xi, z}$ and $r_{n}^{\xi, w}=r_{n}^{\xi, z}$ for $w \in\left(z, z+b_{n}^{\xi}\right)$ we get

$$
\int_{\boldsymbol{A}_{n}^{\xi, z}} f_{n}\left(\mu\left|D_{\xi} u_{n}^{\xi}\right|^{2}\right) \mathrm{d} x=c_{\xi} \int_{z}^{z+b_{n}^{\xi}} F_{n}^{\xi, w}\left(l_{n}^{\xi, w}, r_{n}^{\xi, w}\right) \mathrm{d} w
$$

It follows that

$$
\int_{A} f_{n}\left(\mu\left|D_{\xi} u_{n}^{\xi}\right|^{2}\right) \mathrm{d} x \geqslant \sum_{z \in b_{n}^{\xi} \mathbb{Z}} \int_{\boldsymbol{A}_{n}^{\xi, z}} f_{n}\left(\mu\left|D_{\xi} u_{n}^{\xi}\right|^{2}\right) \mathrm{d} x \geqslant c_{\xi} \int_{Z} F_{n}^{\xi, w}\left(l_{n}^{\xi, w}, r_{n}^{\xi, w}\right) \mathrm{d} w .
$$

In the rest of this section we will prove that

$$
\liminf _{n \rightarrow+\infty} F_{n}^{\xi, w}\left(l_{n}^{\xi, w}, r_{n}^{\xi, w}\right) \geqslant \gamma \eta_{\xi} \#\left(J\left(u^{\xi, w}\right) \cap A^{\xi, w}\right) .
$$

By a standard localization argument, we can assume that $A^{\xi, z}$ is an interval $I=(-k, k)$ and that $J\left(u^{\xi, z}\right)=\{0\}$. Moreover, by an approximation argument, it is not restrictive to assume that $f(t)=$ $t \wedge 1$. Thus, under these conditions, it is sufficient to show that

$$
\liminf _{n \rightarrow+\infty} F_{n}^{\xi, w}\left(l_{n}^{\xi, w}, r_{n}^{\xi, w}\right) \geqslant \eta_{\xi} .
$$

The proof does not rely on the one of the previous section. Indeed, here each term of $F_{n}$ depends both on $l_{n}$ and $r_{n}$, and thus it is not possible to consider separately the left and right contributions. 
As a matter of fact, the proof is based on a suitable control of the difference between $D_{L}^{i}\left(l_{n}, r_{n}\right)$ and $D_{R}^{i}\left(l_{n}, r_{n}\right)$. For this reason, let us define $e_{n}^{i}=r_{n}^{i}-l_{n}^{i}$ (for $-k_{n} \leqslant i \leqslant k_{n}$ ) and denote by $e_{n}$ its piecewise linear interpolation. Clearly $e_{n} \rightarrow 0$ in $L^{1}$. Depending again on whether $Q_{n}^{\xi, z}$ is a square or not we have

$$
h_{n}^{\xi} D_{R}^{i}\left(l_{n}, r_{n}\right)-h_{n}^{\xi} D_{L}^{i}\left(l_{n}, r_{n}\right)=(1-\theta)\left(r_{n}^{i+1}-l_{n}^{i+1}\right)-(1-\theta)\left(r_{n}^{i}-l_{n}^{i}\right)
$$

or

$$
h_{n}^{\xi} D_{R}^{i}\left(l_{n}, r_{n}\right)-h_{n}^{\xi} D_{L}^{i}\left(l_{n}, r_{n}\right)=\theta\left(r_{n}^{i+1}-l_{n}^{i+1}\right)-\theta\left(r_{n}^{i}-l_{n}^{i}\right),
$$

which give respectively

$$
\begin{aligned}
(1-\theta)\left(e_{n}^{i+1}-e_{n}^{i}\right) & =h_{n}^{\xi}\left(D_{R}^{i}\left(l_{n}, r_{n}\right)-D_{L}^{i}\left(l_{n}, r_{n}\right)\right), \\
\theta\left(e_{n}^{i+1}-e_{n}^{i}\right) & =h_{n}^{\xi}\left(D_{R}^{i}\left(l_{n}, r_{n}\right)-D_{L}^{i}\left(l_{n}, r_{n}\right)\right) .
\end{aligned}
$$

For convenience we introduce another interval $I^{\prime}=\left(-k^{\prime}, k^{\prime}\right)$ for $0<k^{\prime}<k$ and define $\bar{e}_{n}=$ $\max \left\{\left|e_{n}^{i}\right|: i h_{n}^{\xi} \in I^{\prime}\right\}$. For a suitable index $j_{n}$ let $y_{n}=j_{n} h_{n}^{\xi}$ be such that $\bar{e}=e\left(y_{n}\right)$. Now we will assume two different cases, according as $\liminf _{n \rightarrow+\infty} \bar{e}_{n}>0$ or $\liminf _{n \rightarrow+\infty} \bar{e}_{n}=0$.

Let us assume first that $\liminf _{n \rightarrow+\infty} \bar{e}_{n}=e^{\prime}>0$. Possibly passing to a subsequence, we can assume that $y_{n} \rightarrow y$ for $y \in\left[-k^{\prime}, k^{\prime}\right]$. Since $e_{n} \rightarrow 0$, for $\delta>0$ and $n$ sufficiently large there exists an index $i_{n}$ such that $y-\delta<i_{n} h_{n}^{\xi}<y$ and $\left|e_{n}^{i_{n}}\right|<\bar{e}_{n} / 2$. Thus from 20, and 21] we can write

$$
\sum_{i=i_{n}}^{j_{n}-1} h_{n}^{\xi}\left|D_{L}^{i}\left(l_{n}, r_{n}\right)-D_{R}^{i}\left(l_{n}, r_{n}\right)\right| \geqslant(\theta \wedge(1-\theta)) \sum_{i=i_{n}}^{j_{n}-1}\left|e_{n}^{i+1}-e_{n}^{i}\right| .
$$

Since the last term gives the variation of $e_{n}$ in $\left(i_{n} h_{n}^{\xi}, j_{n} h_{n}^{\xi}\right)$ we get

$$
\sum_{i=i_{n}}^{j_{n}-1} h_{n}^{\xi}\left|D_{L}^{i}\left(l_{n}, r_{n}\right)-D_{R}^{i}\left(l_{n}, r_{n}\right)\right| \geqslant(\theta \wedge(1-\theta))\left|e_{n}^{i_{n}}-e_{n}^{j_{n}}\right| \geqslant 2 c \bar{e}_{n},
$$

where the constant $c$ depends only on $\theta$. Hence,

$$
2 c \bar{e}_{n} \leqslant \sum_{i=i_{n}}^{j_{n}-1} h_{n}^{\xi}\left|D_{L}^{i}\left(l_{n}, r_{n}\right)-D_{R}^{i}\left(l_{n}, r_{n}\right)\right| \leqslant \sum_{i=i_{n}}^{j_{n}-1} h_{n}^{\xi}\left|D_{L}^{i}\left(l_{n}, r_{n}\right)\right|+\sum_{i=i_{n}}^{j_{n}-1} h_{n}^{\xi}\left|D_{R}^{i}\left(l_{n}, r_{n}\right)\right| .
$$

Therefore one of the two terms on the right hand side is greater than or equal to $c \bar{e}_{n}$. Let us assume it is the first. Now we will show that

$$
\sum_{i=i_{n}}^{j_{n}-1} h_{n}^{\xi}\left|D_{L}^{i}\left(l_{n}, r_{n}\right)\right| \geqslant c^{\prime} \bar{e}_{n}^{2} / \delta
$$

where $c^{\prime}>0$ does not depend on $n$ and $\delta$. Let $L_{n}: I \rightarrow \mathbb{R}$ be a piecewise affine function defined at the knots $i h_{n}^{\xi}$ by

$$
L_{n}^{i}= \begin{cases}0 & \text { for } i \leqslant i_{n} \\ L_{n}^{i-1}+h_{n}^{\xi}\left|D_{L}^{i}\left(l_{n}, r_{n}\right)\right| & \text { for } i_{n}+1 \leqslant i \leqslant j_{n} \\ L_{n}^{j_{n}} & \text { for } i>j_{n} .\end{cases}
$$


Note that $L_{n}^{j_{n}}=\sum_{i=i_{n}}^{j_{n}-1} h_{n}^{\xi}\left|D_{L}^{i}\left(l_{n}, r_{n}\right)\right| \geqslant c \bar{e}_{n}$. Thus

$$
\sum_{i=i_{n}}^{j_{n}-1} \frac{1}{2} h_{n}^{\xi}\left|D_{L}^{i}\left(l_{n}, r_{n}\right)\right|^{2} \geqslant \frac{1}{2} \int_{I}\left|L_{n}^{\prime}\right|^{2} \mathrm{~d} y \geqslant c^{\prime} \bar{e}_{n}^{2} / \delta .
$$

Now we can show that for $n$ sufficiently large we have

$$
\sum_{i=i_{n}}^{j_{n}-1} \frac{1}{2} h_{n}^{\xi} f_{n}\left(\mu\left|D_{L}^{i}\left(l_{n}, r_{n}\right)\right|^{2}\right) \geqslant \frac{1}{2} \eta_{\xi}
$$

This inequality is obvious if, for some index $i$, we have $h_{n} \mu\left|D_{L}^{i}\left(l_{n}, r_{n}\right)\right|^{2}>1$; indeed, as $f(t)=$ $t \wedge 1$ we then have

$$
f_{n}\left(\mu\left|D_{L}^{i}\left(l_{n}, r_{n}\right)\right|^{2}\right)=\left(1 / h_{n}\right) f\left(\mu h_{n}\left|D_{L}^{i}\left(l_{n}, r_{n}\right)\right|^{2}\right)=1 / h_{n},
$$

which gives 24) since $\eta_{\xi}=h_{n}^{\xi} / h_{n}$. Otherwise we have $h_{n} \mu\left|D_{L}^{i}\left(l_{n}, r_{n}\right)\right|^{2}<1$ for every index $i$ and then by 23 ,

$$
\sum_{i=i_{n}}^{j_{n}-1} \frac{1}{2} h_{n}^{\xi} f_{n}\left(\mu\left|D_{L}^{i}\left(l_{n}, r_{n}\right)\right|^{2}\right) \geqslant \frac{1}{2} \mu \sum_{i=i_{n}}^{j_{n}-1} h_{n}^{\xi}\left|D_{L}^{i}\left(l_{n}, r_{n}\right)\right|^{2} \geqslant c^{\prime} \bar{e}_{n}^{2} / \delta
$$

As $\bar{e}_{n} \rightarrow e^{\prime}>0$, for $\delta$ sufficiently small we get the required inequality.

In order to deduce (19) from (24) it is sufficient to remark that, by symmetry, we can apply the same reasoning for another index $i_{n}^{\prime}$ such that $y<i_{n}^{\prime} h_{n}^{\xi}<y+\delta$.

Now, let us consider the case $\liminf _{n \rightarrow+\infty} \bar{e}_{n}=0$. For $\delta>0$ we define

$$
g_{n}^{\delta}(t)= \begin{cases}|t| / \delta, & |t|<\delta / h_{n}, \\ 1 / h_{n}, & \text { otherwise. }\end{cases}
$$

It is easy to see that $g_{n}^{\delta}(t) \leqslant f_{n}\left(t^{2}\right)+1 / \delta^{2}$. We recall that $J\left(u^{\xi, z}\right)=\{0\}$ and we denote by $\left[u^{\xi, z}\right]$ its jump. For $\delta>0$ let $j_{n}>0$ be such that $j_{n} h_{n}^{\xi} \searrow \delta^{3}$. Then for $n \gg 1$ and $\mu^{\prime}=\sqrt{\mu}$ we get

$$
\begin{aligned}
F_{n}^{\xi, z}\left(l_{n}^{\xi, z}, r_{n}^{\xi, z}\right) & \geqslant \sum_{i=-j_{n}}^{j_{n}-1}\left(\frac{1}{2} h_{n}^{\xi} f_{n}\left(\mu\left|D_{L}^{i}\left(l_{n}, r_{n}\right)\right|^{2}\right)+\frac{1}{2} h_{n}^{\xi} f_{n}\left(\mu\left|D_{R}^{i}\left(l_{n}, r_{n}\right)\right|^{2}\right)\right) \\
& \geqslant \sum_{i=-j_{n}}^{j_{n}-1}\left(\frac{1}{2} h_{n}^{\xi} g_{n}^{\delta}\left(\mu^{\prime} D_{L}^{i}\left(l_{n}, r_{n}\right)\right)+\frac{1}{2} h_{n}^{\xi} g_{n}^{\delta}\left(\mu^{\prime} D_{R}^{i}\left(l_{n}, r_{n}\right)\right)\right)-3 \delta .
\end{aligned}
$$

Now we distinguish between two cases. First we assume that for every index $i=-j_{n}, \ldots, j_{n}-1$ we have $\left|\mu^{\prime} D_{L}^{i}\left(l_{n}, r_{n}\right)\right| \wedge\left|\mu^{\prime} D_{R}^{i}\left(l_{n}, r_{n}\right)\right| \leqslant \delta / h$. Then

$$
F_{n}^{\xi, z}\left(l_{n}^{\xi, z}, r_{n}^{\xi, z}\right) \geqslant \frac{c}{\delta} \sum_{i=-j_{n}}^{j_{n}-1}\left(h_{n}^{\xi}\left|D_{L}^{i}\left(l_{n}, r_{n}\right)\right|+h_{n}^{\xi}\left|D_{R}^{i}\left(l_{n}, r_{n}\right)\right|\right)-3 \delta .
$$


Remembering that $D_{L}^{i}\left(l_{n}, r_{n}\right)=D_{\xi} u_{n}^{\xi, z}$, and similarly for $D_{R}^{i}\left(l_{n}, r_{n}\right)$, we can write

$$
F_{n}^{\xi, z}\left(l_{n}^{\xi, z}, r_{n}^{\xi, z}\right) \geqslant \frac{c}{\delta} \sum_{i=-j_{n}}^{j_{n}-1} h_{n}^{\xi}\left|D_{\xi} u_{n}^{\xi, z}\right|-3 \delta \geqslant \frac{c}{\delta} \int_{-\delta}^{\delta}\left|\left(u_{n}^{\xi, z}\right)^{\prime}\right| \mathrm{d} y-3 \delta .
$$

By the lower semicontinuity of the total variation (with respect to the $L^{1}$-topology), we get

$$
\liminf _{n \rightarrow+\infty} F_{n}^{\xi, z}\left(l_{n}^{\xi, z}, r_{n}^{\xi, z}\right) \geqslant \frac{c}{\delta}\left|\left[u^{\xi, z}\right]\right|-3 \delta,
$$

which gives 19 for $\delta$ sufficiently small.

Now we assume that for some index $i$ we have $\left|\mu^{\prime} D_{L}^{i}\left(l_{n}, r_{n}\right)\right| \wedge\left|\mu^{\prime} D_{R}^{i}\left(l_{n}, r_{n}\right)\right|>\delta / h$. Then, $g_{n}^{\delta}$ being Lipschitz continuous with constant $1 / \delta$, by (20) and (21) we can write

$$
\left|g_{n}^{\delta}\left(\mu^{\prime} D_{L}^{i}\left(l_{n}, r_{n}\right)\right)-g_{n}^{\delta}\left(\mu^{\prime} D_{R}^{i}\left(l_{n}, r_{n}\right)\right)\right| \leqslant \frac{1}{\delta}\left|\mu^{\prime} D_{L}^{i}\left(l_{n}, r_{n}\right)-\mu^{\prime} D_{R}^{i}\left(l_{n}, r_{n}\right)\right| \leqslant c \bar{e}_{n} / h_{n}^{\xi},
$$

where $c$ depends on $\delta, \theta$ and $\mu$. Then, assuming for instance that $\left|\mu^{\prime} D_{R}^{i}\left(l_{n}, r_{n}\right)\right| \geqslant \delta / h$ we get $g_{n}^{\delta}\left(\mu^{\prime} D_{R}^{i}\left(l_{n}, r_{n}\right)\right)=1 / h_{n}$, and so

$$
g_{n}^{\delta}\left(\mu^{\prime} D_{L}^{i}\left(l_{n}, r_{n}\right)\right)+g_{n}^{\delta}\left(\mu^{\prime} D_{R}^{i}\left(l_{n}, r_{n}\right)\right) \geqslant 2 / h_{n}-c \bar{e}_{n} / h_{n}^{\xi}
$$

It follows that

$$
\frac{1}{2} h_{n}^{\xi} g_{n}^{\delta}\left(\mu\left|D_{L}^{i}\left(l_{n}, r_{n}\right)\right|^{2}\right)+\frac{1}{2} h_{n}^{\xi} g_{n}^{\delta}\left(\mu\left|D_{L}^{i}\left(l_{n}, r_{n}\right)\right|^{2}\right) \geqslant \frac{1}{2} h_{n}^{\xi}\left(2 / h_{n}-c \bar{e}_{n} / h_{n}^{\xi}\right)=\eta_{\xi}-c^{\prime} \bar{e}_{n} .
$$

As $\bar{e}_{n} \rightarrow 0$ we get the right inequality.

\subsection{Estimate of the surface energy}

Now we can complete the proof of the $\Gamma$-liminf inequality for the length energy. In the previous two subsections we have shown that 15 holds true for every sequence $u_{n} \in V_{n}$ converging to $u$ in $L^{1}(\Omega)$. Thus, for every $\xi=\zeta /|\zeta|$ with $\zeta \in \mathbb{Z}^{2}$, we have

$$
\Gamma-\liminf _{n \rightarrow+\infty} G_{n}(u, A) \geqslant \gamma \int_{J \xi(u) \cap A} \eta_{\xi}|\langle\nu, \xi\rangle| \mathrm{d} s=\gamma \int_{J(u) \cap A} \psi_{\xi} \mathrm{d} s,
$$

where

$$
\psi_{\xi}(x)= \begin{cases}\eta_{\xi}|\langle\nu, \xi\rangle| & \text { if } x \in J^{\xi}(u), \\ 0 & \text { otherwise. }\end{cases}
$$

For $\mathrm{d} \lambda=\mathrm{d} s$ and $\mu(A)=\Gamma-\liminf _{n \rightarrow+\infty} G_{n}(u, A)$, by Proposition A.4 we are allowed to take in 25 the pointwise supremum of the family $\psi_{\xi}$. Hence it will be sufficient to show that $\sup _{\xi} \psi_{\xi}(x) \geqslant$ $\phi(v)$. For $x \in J(u)$ let $v=v(x)$ be the normal to $J(u)$ and let $\phi(v)=\tau_{i}\left|\left\langle v, \xi_{i}\right\rangle\right|$ for an index $i=1, \ldots, 3$. Let $u^{+}=u^{+}(x)$ and $u^{-}=u^{-}(x)$ be the traces of $u$ on $J(u)$. If $\left\langle u^{+}-u^{-}, \xi_{i}\right\rangle \neq 0$ then $x \in J^{\xi_{i}}(u)$ and $\psi_{\xi_{i}}(x)=\eta_{\xi_{i}}\left|\left\langle v, \xi_{i}\right\rangle\right|=\tau_{i}\left|\left\langle v, \xi_{i}\right\rangle\right|$. Obviously $\sup _{\xi} \psi_{\xi}(x) \geqslant \tau_{i}\left|\left\langle v, \xi_{i}\right\rangle\right|=\phi(v)$. On the contrary, if $\left\langle u^{+}-u^{-}, \xi_{i}\right\rangle=0$ then $\psi_{\xi}(x)=0$. In this case let us take a sequence $\xi^{k}$ which converges to $\xi_{i}$ with $\xi^{k} \neq \xi_{i}$ for every $k \in \mathbb{N}$. As $u^{+}-u^{-} \neq 0$ and the vectors $\xi^{k}$ and $\xi_{i}$ are independent, it follows that $\left\langle u^{+}-u^{-}, \xi^{k}\right\rangle \neq 0$. Therefore $x \in J^{\xi^{k}}(u)$ and $\psi_{\xi^{k}}(x)=\eta_{\xi^{k}}\left|\left\langle v, \xi^{k}\right\rangle\right|$. Since $\eta_{\xi}=h_{n}^{\xi} / h_{n}$, by the definition of the height $h_{n}^{\xi}$ it is clear that $\eta_{\xi^{k}}$ converges to $\eta_{\xi_{i}}=\tau_{i}$ (see also Figure 5. Then $\sup _{\xi} \psi_{\xi}(x) \geqslant \sup _{k} \psi_{\xi^{k}}(x) \geqslant \tau_{i}\left|\left\langle\nu, \xi_{i}\right\rangle\right|=\phi(\nu)$. 


\section{A. Some technical results}

\section{A.1 Basic facts about the space SBD}

In this appendix we recall briefly some results about the space $S B D$ of special functions with bounded deformation. We will always assume that $\Omega$ is an open, bounded, Lipschitz set in $\mathbb{R}^{2}$. A vector field $u \in L^{1}\left(\Omega, \mathbb{R}^{2}\right)$ belongs to $S B D(\Omega)$ if the symmetrized distributional derivative $E u=\frac{1}{2}\left(D u+D u^{T}\right)$ is a finite measure which can be written as

$$
E u=\varepsilon(u) \mathcal{L}^{n}+\left(u^{+}-u^{-}\right) \odot v_{u} \mathcal{H}^{1}\llcorner J(u) .
$$

For simplicity we will denote by $d x$ the Lebesgue measure and by $d s$ the one-dimensional Hausdorff measure. When $\varepsilon(u) \in L^{p}\left(\Omega, \mathbb{R}^{2 \times 2}\right)$ for $p>1$ and $\mathcal{H}^{1}(J(u))<+\infty$ we will say that $u \in$ $S B D^{p}(\Omega)$.

The following compactness and lower semicontinuity result is proved in [5]; the extension to anisotropic energies can be found in [23].

Proposition A.1 Let $\phi$ be a norm in $\mathbb{R}^{2}$ and $u_{k}$ be a sequence in $S B D^{2}(\Omega)$ such that

$$
\int_{\Omega \backslash J\left(u_{k}\right)} W^{e}\left(\varepsilon\left(u_{k}\right)\right) \mathrm{d} x+\mathcal{H}^{1}\left(J\left(u_{k}\right)\right)+\left\|u_{k}\right\|_{\infty} \leqslant C .
$$

Then there exist a subsequence $u_{n}$ of $u_{k}$ and a function $u \in S B D^{2}(\Omega)$ such that $u_{n} \rightarrow u$ in $L^{1}\left(\Omega, \mathbb{R}^{2}\right), \varepsilon\left(u_{n}\right) \rightarrow \varepsilon(u)$ in $L^{1}\left(\Omega, \mathbb{R}^{2 \times 2}\right)$ and

$$
\int_{J(u)} \phi(v) \mathrm{d} s \leqslant \liminf _{n \rightarrow+\infty} \int_{J\left(u_{n}\right)} \phi(v) \mathrm{d} s .
$$

For $\xi \in \mathbb{S}^{1}$ we denote by $Z$ the subspace $\left\langle\xi^{\perp}\right\rangle$ generated by $\xi^{\perp}$ and by $J^{\xi}(u)$ the subset of $J(u)$ where $\left\langle u^{+}-u^{-}, \xi\right\rangle \neq 0$. For $z \in Z$ let $\Omega^{\xi, z}$ be the section $\{x \in \Omega: x=z+t \xi$ for $t \in \mathbb{R}\}$ and let $u^{\xi, z}$ be the (scalar) function

$$
u^{\xi, z}(t)=\langle u(z+t \xi), \xi\rangle
$$

The functions in $S B D(\Omega)$ have the following slicing properties [2].

Proposition A.2 Let $u \in S B D(\Omega)$. For a.e. $\xi \in \mathbb{S}^{1}$ and for a.e. $z \in Z$ the function $u^{\xi, z}$ belongs to $\operatorname{SBV}\left(\Omega^{\xi, z}\right)$. Moreover, for every open set $B \subset \Omega$ we have

$$
\int_{Z} \mathcal{H}^{0}\left(J\left(u^{\xi, z}\right) \cap B^{\xi, z}\right) \mathrm{d} z=\int_{J^{\xi}(u) \cap B}|\langle v, \xi\rangle| \mathrm{d} s .
$$

The $\Gamma$-limsup inequality is based on the following density result, which follows from [12] and Reshentyak's theorem (see e.g. [3, Theorem 2.39]). (It is enough to check that the sequence $u_{k}$ defined in [12] satisfies $v_{k} \mathcal{H}^{1}\left\llcorner J\left(u_{k}\right) \stackrel{\star}{\rightarrow} \nu \mathcal{H}^{1}\llcorner J(u)\right.$ in the sense of measures.)

Proposition A.3 Let $\phi$ be a norm in $\mathbb{R}^{2}$. Then for every $u \in S B D^{2}(\Omega) \cap L^{\infty}\left(\Omega, \mathbb{R}^{2}\right)$ there exists a sequence $u_{n}$ in $S B D^{2}(\Omega)$ such that $J\left(u_{n}\right)$ is a finite union of disjoint segments, $u_{n} \in$ $W^{k, \infty}\left(\Omega, \mathbb{R}^{2}\right)$ (for $k$ arbitrarily large), $\left\|u_{n}\right\|_{\infty} \leqslant\|u\|_{\infty}$ and

$$
\begin{gathered}
u_{n} \rightarrow u \quad \text { in } L^{1}\left(\Omega, \mathbb{R}^{2}\right), \quad \varepsilon\left(u_{n}\right) \rightarrow \varepsilon(u) \quad \text { in } L^{2}\left(\Omega, \mathbb{R}^{2 \times 2}\right), \\
\lim _{n \rightarrow+\infty} \int_{J\left(u_{n}\right)} \phi(v) \mathrm{d} s \leqslant \int_{J(u)} \phi(v) \mathrm{d} s .
\end{gathered}
$$




\section{A.2 The supremum of a family of measures}

This measure-theoretic result, proved e.g. in [8], is used for the $\Gamma$-liminf inequality.

Proposition A.4 Denote by $\mathcal{A}(\Omega)$ the collection of open sets contained in $\Omega$. Let $\mu: \mathcal{A}(\Omega) \rightarrow$ $[0,+\infty)$ be superadditive on open sets with disjoint compact closures and let $\lambda$ be a positive Borel measure in $\Omega$. Let $\psi_{i}$ be a family of positive Borel functions such that

$$
\int_{A} \psi_{i} \mathrm{~d} \lambda \leqslant \mu(A) \quad \text { for all } A \in \mathcal{A}(\Omega)
$$

Then

$$
\int_{A} \sup _{i} \psi_{i}(x) \mathrm{d} \lambda \leqslant \mu(A) \quad \text { for all } A \in \mathcal{A}(\Omega) .
$$

\section{Acknowledgements}

This work has been partially supported by Istituto Nazionale di Alta Matematica through the grant "Mathematical Challenges in Nanomechanics".

\section{REFERENCES}

1. Alicandro, R., Focardi, M., \& Gelli, M. S. Finite-difference approximation of energies in fracture mechanics. Ann. Scuola Norm. Sup. Pisa Cl. Sci. (4) 29 (2000), 671-709. Zbl 1072.49020 MR 1817714

2. Ambrosio, L., Coscia, A., \& Dal MAso, G. Fine properties of functions with bounded deformation. Arch. Ration. Mech. Anal. 139 (1997), 201-238. Zbl 0890.49019 MR 1480240

3. Ambrosio, L., Fusco, N., \& Pallara, D. Functions of Bounded Variation and Free Discontinuity Problems. Oxford Math. Monogr., Oxford Univ. Press, New York (2000). Zbl $0890.49019 \mid$ MR 1857292

4. BAŽANT, Z. P., \& LIN, F. B. Nonlocal smeared cracking model for concrete fracture. J. Structural Engrg. 114 (1988), 2493-2510

5. Bellettini, G., Coscia, A., \& Dal Maso, G. Compactness and lower semicontinuity properties in $\operatorname{SBD}(\Omega)$. Math. Z. 228 (1998), 337-351. Zbl 0914.46007 MR 1630504

6. Bourdin, B., \& Chambolle, A. Implementation of an adaptive finite-element approximation of the Mumford-Shah functional. Numer. Math. 85 (2000), 609-646. Zbl 0961.65062 MR 1771782

7. Bourdin, B., FrAnCFORT, G. A., \& MARIGO, J. J. Numerical experiments in revisited brittle fracture. J. Mech. Phys. Solids 48 (2000), 797-826. Zbl 0995.74057 MR 1745759

8. Braides, A. Approximation of Free-Discontinuity Problems, Lecture Notes in Math. 1694, Springer, Berlin (1998). Zbl 0909.49001 MR 1651773

9. Braides, A., \& DAl MAso, G. Non-local approximation of the Mumford-Shah functional. Calc. Var. Partial Differential Equations 5 (1997), 293-322. Zbl 0873.49009 MR 1450713

10. Сhambolle, A. Image segmentation by variational methods: Mumford and Shah functional and the discrete approximations. SIAM J. Appl. Math. 55 (1995), 827-863. Zbl 0830.49015 MR 1331589

11. Chambolle, A. A density result in two-dimensional linearized elasticity, and applications. Arch. Ration. Mech. Anal. 167 (2003), 211-233. Zbl 1030.74007 MR 1978582

12. Chambolle, A. An approximation result for special functions with bounded deformation. J. Math. Pures Appl. (9) 83 (2004), 929-954. Zbl pre02118455| MR 2074682

13. Chambolle, A., \& Dal Maso, G. Discrete approximation of the Mumford-Shah functional in dimension two. M2AN Math. Model. Numer. Anal. 33 (1999), 651-672. Zbl 0943.49011 MR 1726478 
14. Cortesani, G., \& Toader, R. A density result in SBV with respect to non-isotropic energies. Nonlinear Anal. 38 (1999), 585-604. Zbl 0939.49024 MR 1709990

15. Dal Maso, G. An Introduction to $\Gamma$-Convergence. Progr. Nonlinear Differential Equations Appl. 8, Birkhäuser, Boston, MA (1993). Zbl 0816.49001 MR 1201152

16. Dal Maso, G., Francfort, G. A., \& ToAder, R. Quasistatic crack growth in nonlinear elasticity. Arch. Ration. Mech. Anal. 176 (2005), 165-225. Zbl 1064.74150 MR 2186036

17. Francfort, G. A., \& MARIgo, J. J. Revisiting brittle fracture as an energy minimization problem. J. Mech. Phys. Solids 46 (1998), 1319-1342. Zbl 0966.74060 MR 1633984

18. GRASSL, P., \& JiRÁSEK, M. On mesh bias of local damage models for concrete. Fracture Mechanics of Concrete Structures (FRAMCOS-5, Vail, CO, 2004), 255-262.

19. JiRÁseK, M. Nonlocal models for damage and fracture: comparison of approaches. Internat. J. Solids Structures 35 (1998), 4133-4145. Zbl 0930.74054 MR 1638122

20. Lussardi, L., \& Negri, M. Convergence of non-local finite element energies for fracture mechanics. Numer. Funct. Anal. Optim. 28 (2007), 83-109.

21. Mosler, J., \& Meschke, G. Embedded crack vs. smeared crack models: a comparison of elementwise discontinuous crack path approaches with emphasis on mesh bias. Comput. Methods Appl. Mech. Engrg. 193 (2004), 3351-3375. Zbl 1060.74606

22. NegRi, M. The anisotropy introduced by the mesh in the finite element approximation of the MumfordShah functional. Numer. Funct. Anal. Optim. 20 (1999), 957-982. Zbl 0953.49024 MR 1728172

23. NegRI, M. A finite element approximation of the Griffith's model in fracture mechanics. Numer. Math. 95 (2003), 653-687. Zbl 1068.74080 MR 2013123

24. Negri, M. A non-local approximation of free discontinuity problems in $S B V$ and $S B D$. Calc. Var. Partial Differential Equations 25 (2006), 33-62. Zbl pre05009624 MR 2183854

25. Oliver, J. A consistent characteristic length for smeared cracking models. Int. J. Numer. Methods Engrg. 28 (1989), 461-474. Zbl 0676.73066

26. Ortiz, M., \& Giannakopoulos, K. Crack propagation in monolithic ceramics under mixed mode loading. Internat. J. Fracture 44 (1990), 233-258.

27. RASHID, Y. R. Analysis of prestressed concrete pressure vessels. Nuclear Engrg. Design 7 (1968), 334 344.

28. Weihe, S., KröPlin, B., \& DE Borst, R. Classification of smeared crack models based on material and structural properties. Internat. J. Solids Structures 35 (1998), 1289-1308. Zbl 0932.74005 